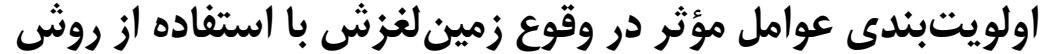

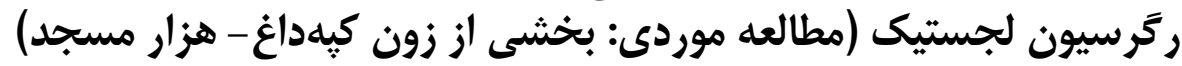

شيوا عبدى بسطامى'، هادى معماريان '، سيّد محمّد تاجبخش 'و محمود اعظمىراد؟ّ

ا - دانشجوى كارشناسى ارشد مهندسى آبخيزدارى دانشخاه بيرجند، (نويسنده مسوول: Bastamish22@ gmail.com)

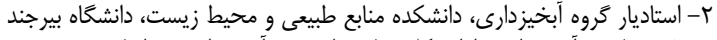

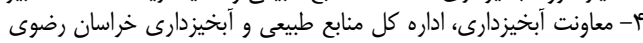

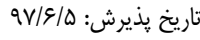

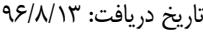

IV. • صفحه:

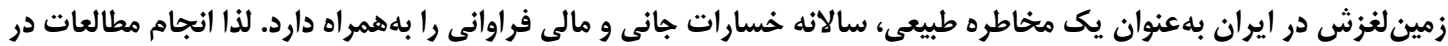

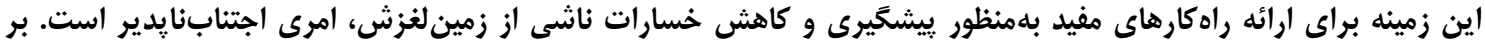

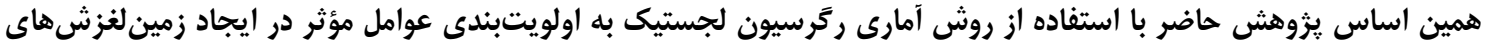

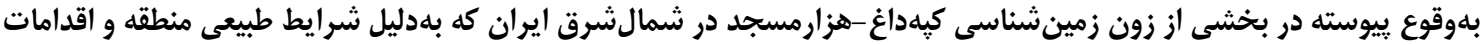

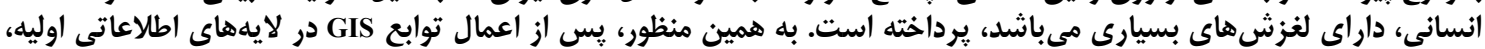

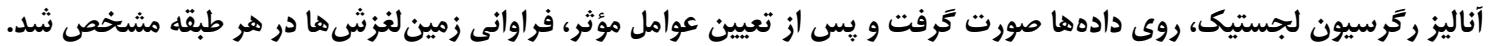

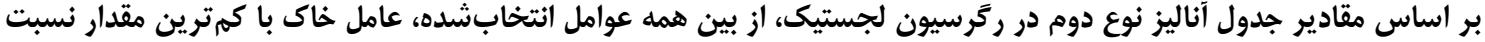
Pr> LR

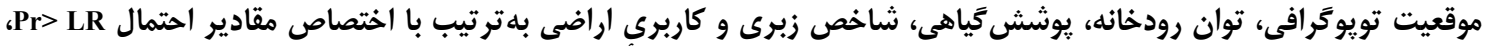

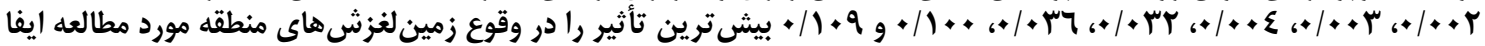

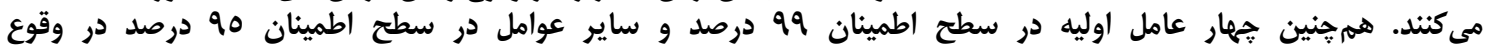
زمين لغزش هاى منطقه مورد مطالعه مؤثر شناخته شدند.

وازههاى كليدى: اولويتبندى، ركر سيون لجستيك، زمينلغزش، سامانه اطلاعات جغرافيايى

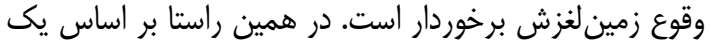

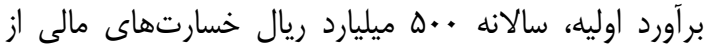

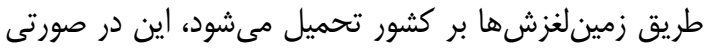

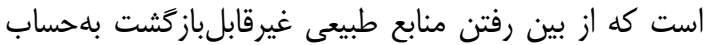

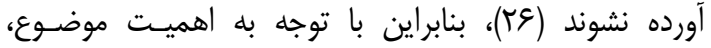

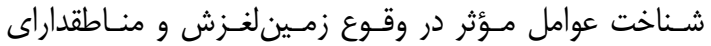

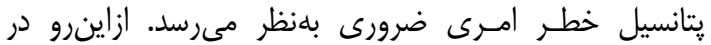

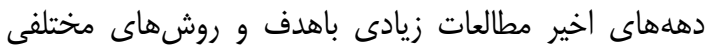

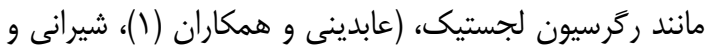

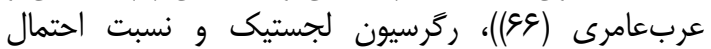

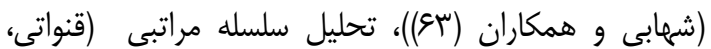

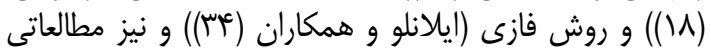

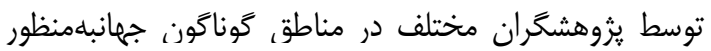

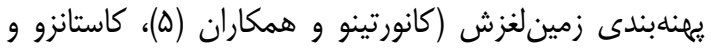

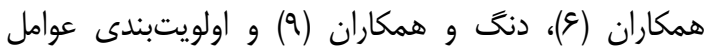

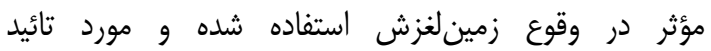

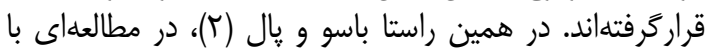

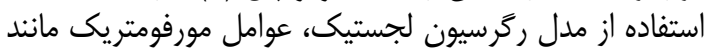

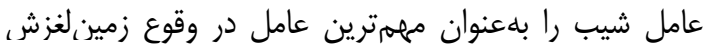

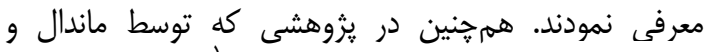

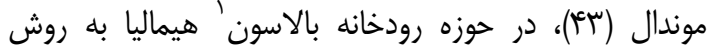

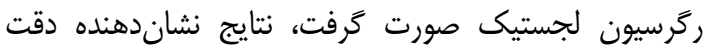
بالاى مدل ركر سيون لجستيك و مناسب و كارئ آمد بودن اين
مقدمه انسان همواره با مسائل محيط زيستى فراوانى مواجه است

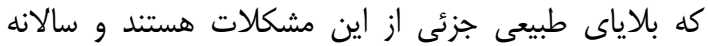

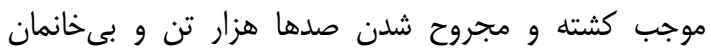

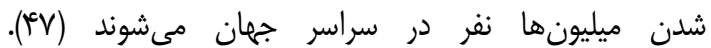

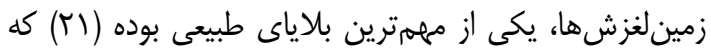

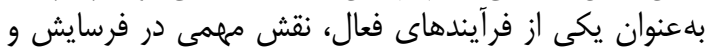

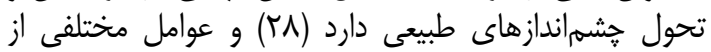

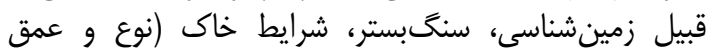

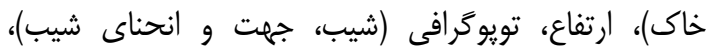

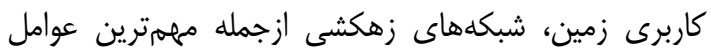

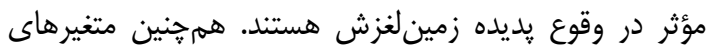

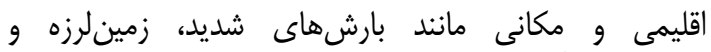

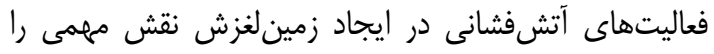

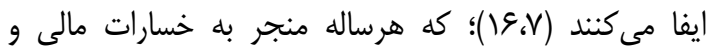

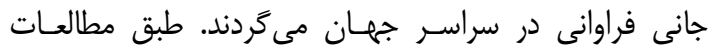

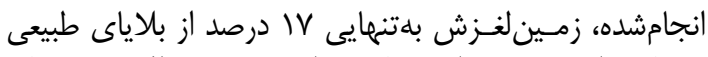

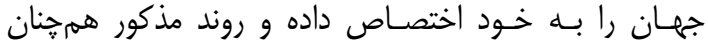

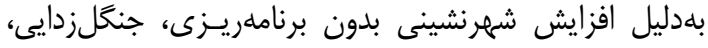

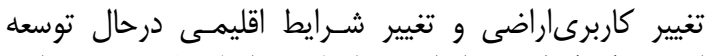

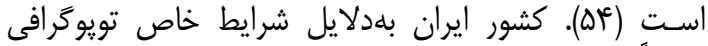

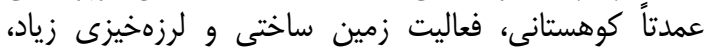

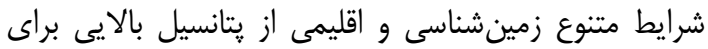


مواد و روشهانها

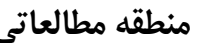

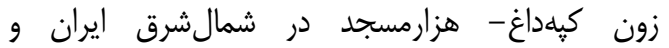

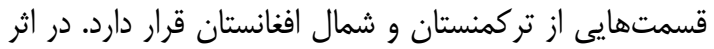

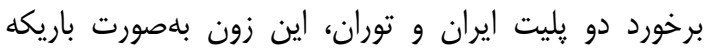

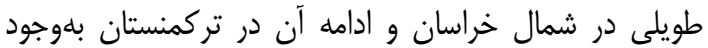

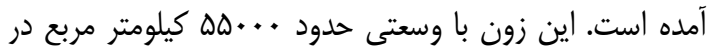

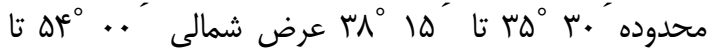

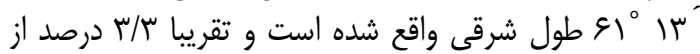

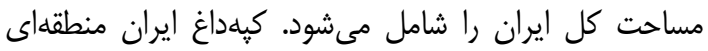

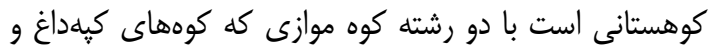
هزار مسجد در شمال و كوههاى كلستان، آلاداغ و بينالود در دران

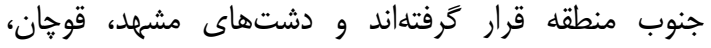

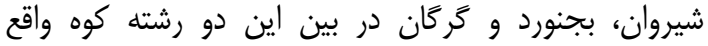

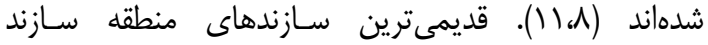

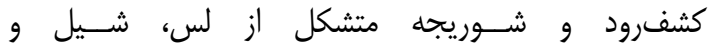

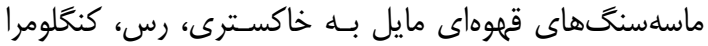

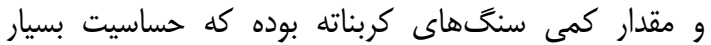

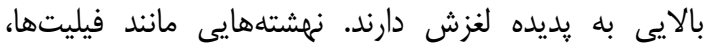

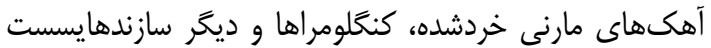

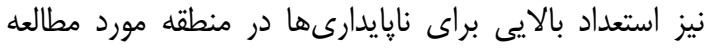

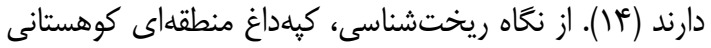

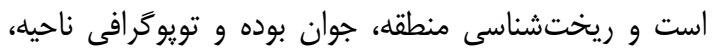

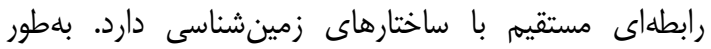

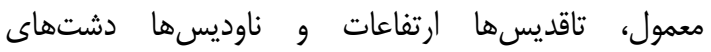

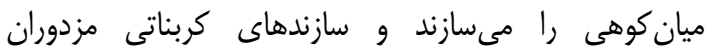

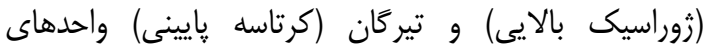

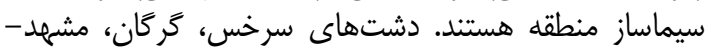

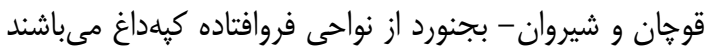

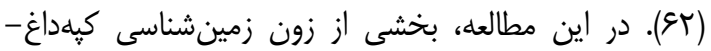

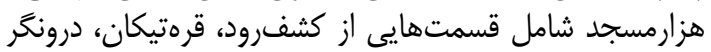

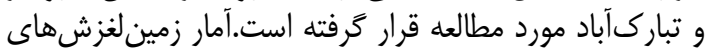

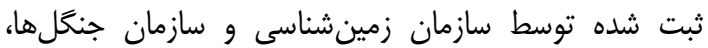

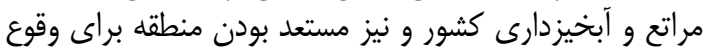

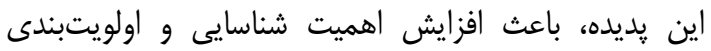

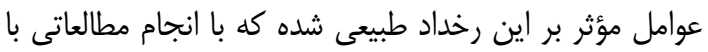

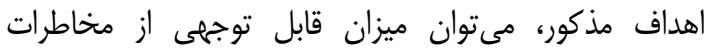
حاصل از وقوع زمينلغزش را كاهش دان ماد.

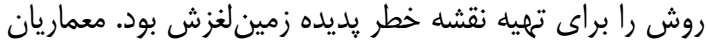

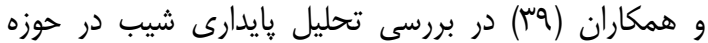

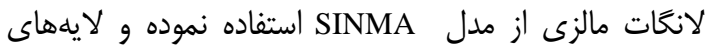

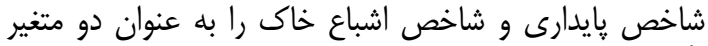

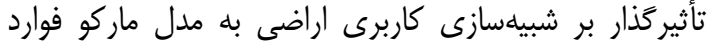

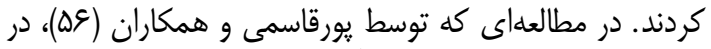

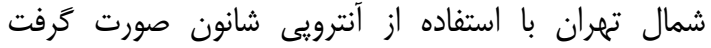

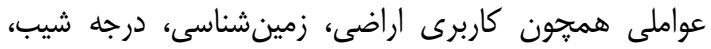

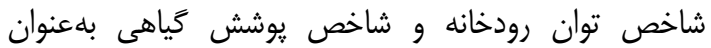

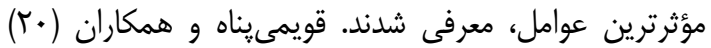

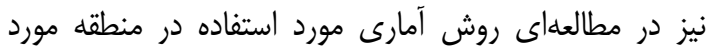

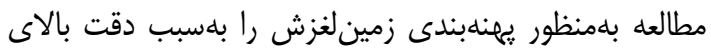

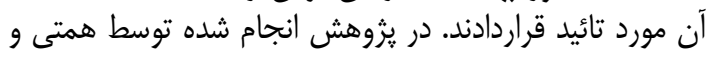

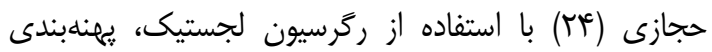

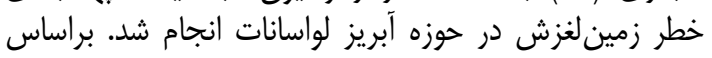

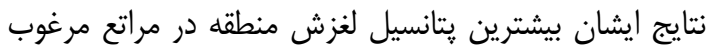

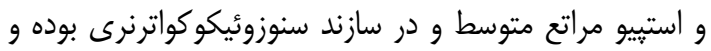

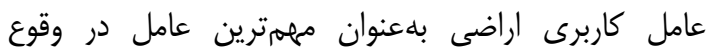
لغزش هاى منطقه معرفى كرديد.

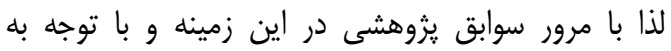

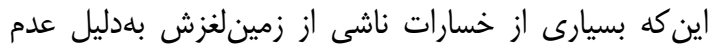

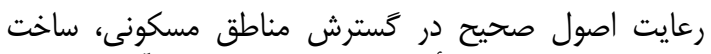

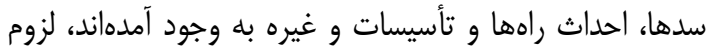

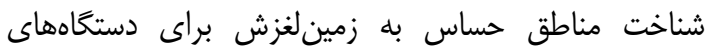

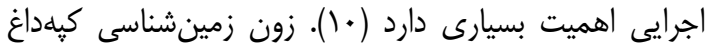

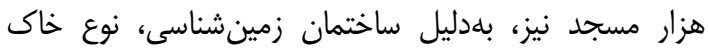

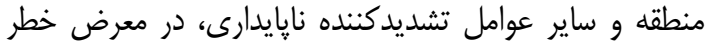

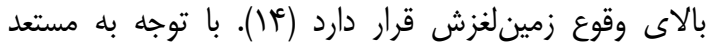

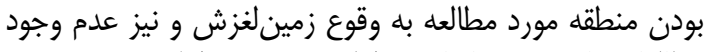

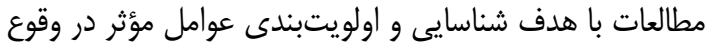

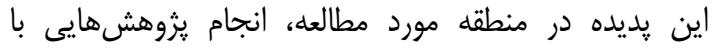

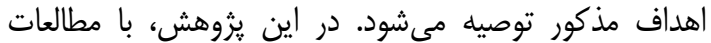

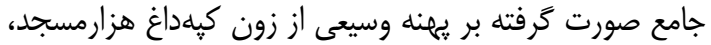

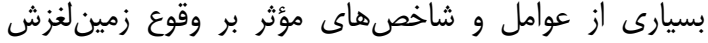

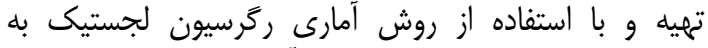

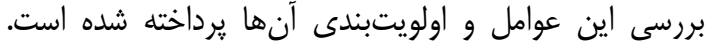

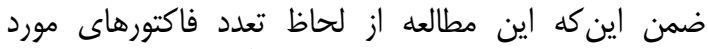
استفاده و سطح وسيع مطالعاتى داراى نوآورى مى إناشد. 


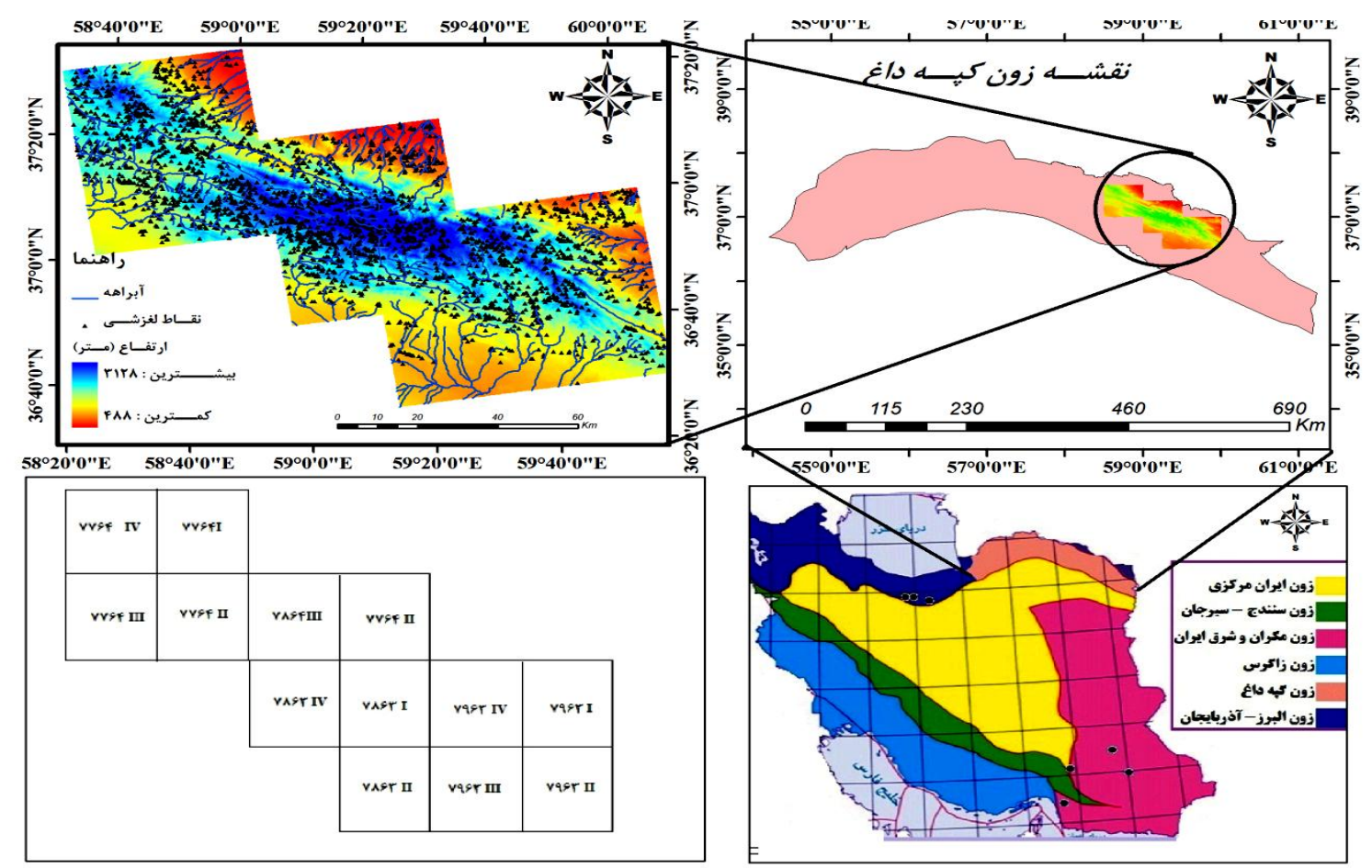

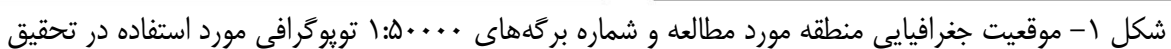

Figure 1.Geographical location of the study area sheet number 1: 50000 topography used in the research

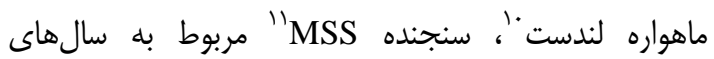
19VT-191VV

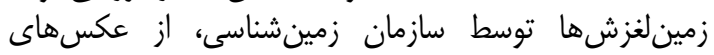

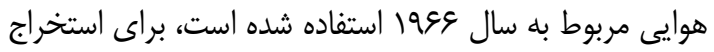

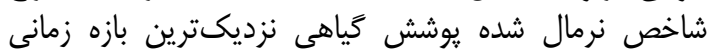

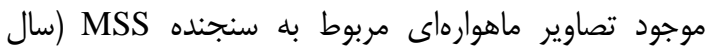

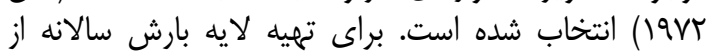

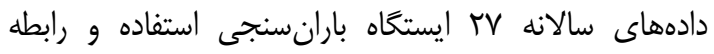

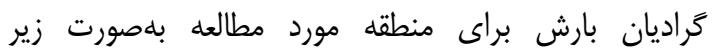

$$
\mathrm{P}=\cdot / \cdot V \Delta S(\mathrm{H})+\mid V V / 9 \quad \mathrm{r}^{\top}=\cdot / \Delta q \mu
$$

كه در آن P ميزان بارندگى سالانه (ميلىمتر) و H ارتفاع (متر) است.

نقشههاى مربوط به عوامل مؤثر از قبيل لايه زبرى، لايه

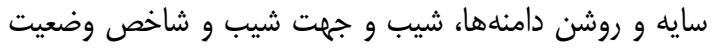

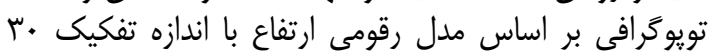

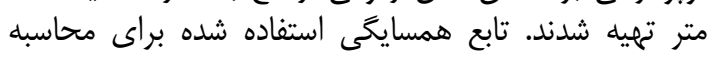

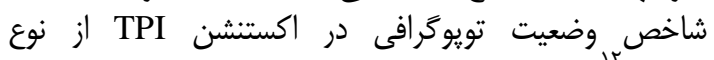

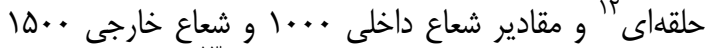

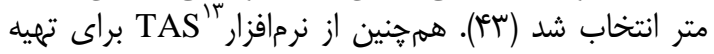

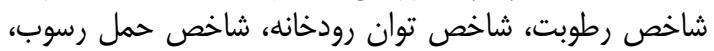

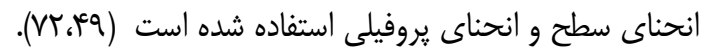

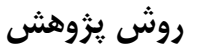

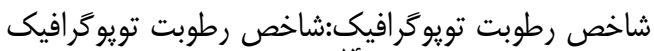

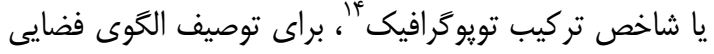

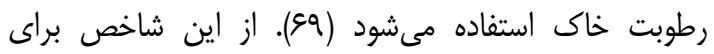

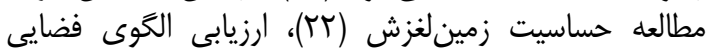

معيارها و عوامل محتمل بر وقوع زمين لغزشهادهاى منطقه

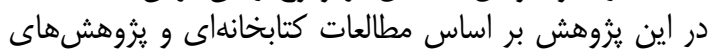

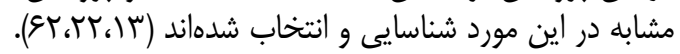

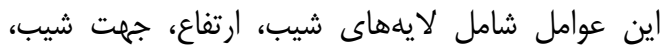

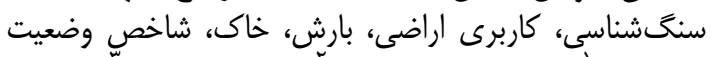

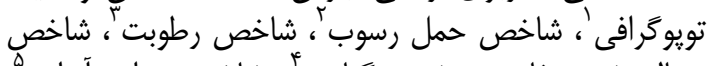

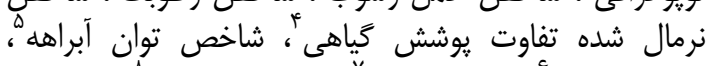

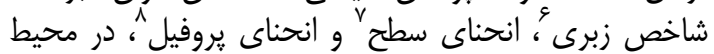

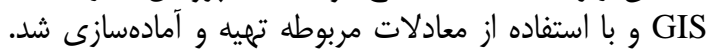

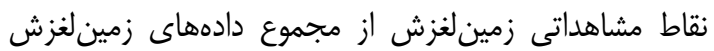

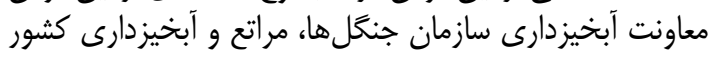

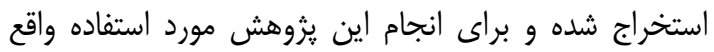

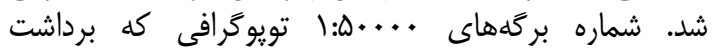

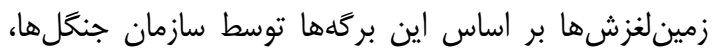

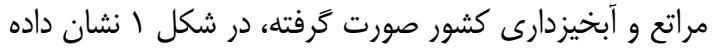

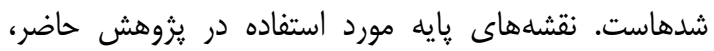

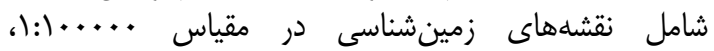

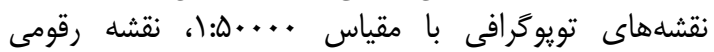

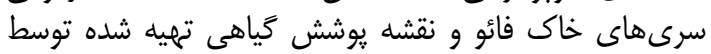

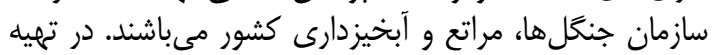

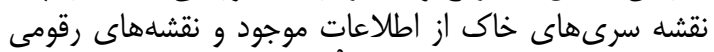
فائو در قالب نرمافزار

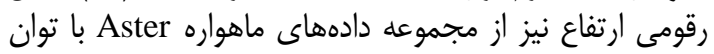

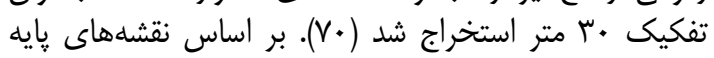

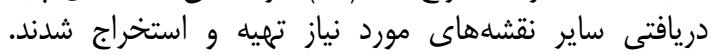
همجنين بلمنظور استخراج شاخص يوشش كَياهى تصاوير نياز

1- Topographic Position Index (TPI) 2- Sediment Transport Index (STI) 3- Topographic Wetness Index (TWI) 4- Normalized Difference Vegetation Index (NDVI) 5- Stream Power Index (SPI) 6- Roughness Index 7- Plan Curvature

10- Landsat

8- Profile Curvature9- Harmonized World Soil Database

13- Terrain Analysis System

11- Multi Spectral Scanner 12-Annulus

14- Compound Topographic Index (CTI) 


\section{شاخص بوشش تَياهى}

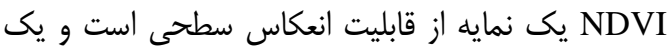

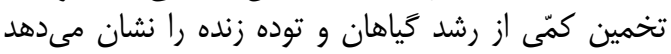

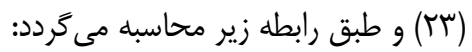

$$
N D V I=(N I R-R) /(N I R+R)
$$

در رابطه فوق، NIR طولموج مادونقرمز نزديك،

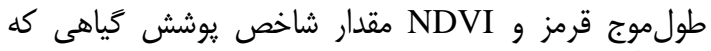

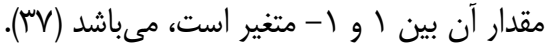

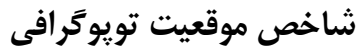

اين شاخص راهكارى نوين جهت تعرئ تعيين كمى لندفرمهافهاه

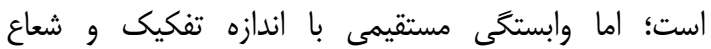

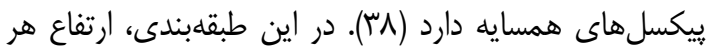

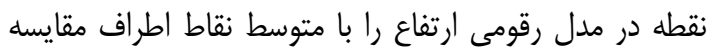

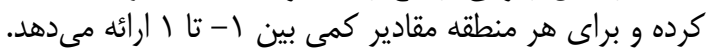

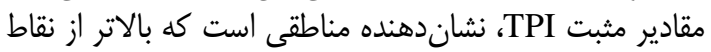

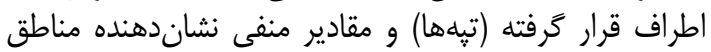

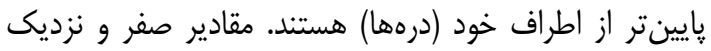

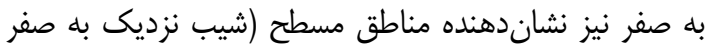

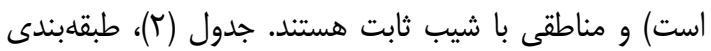

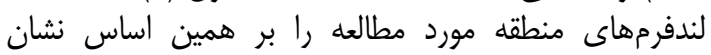

$\mathrm{TPI}=\mathrm{Z}_{0}-\frac{\sum_{\mathrm{n}-1} \mathrm{Z}_{\mathrm{n}}}{\mathrm{n}}$

در رابطه فوق؛

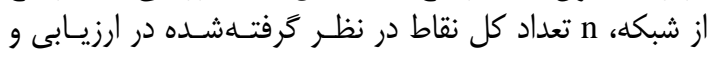

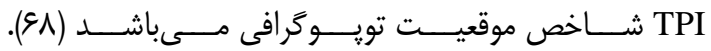

\begin{tabular}{|c|c|c|}
\hline مقدار TPI & 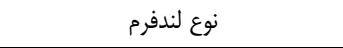 & توصيفات \\
\hline$-1<\mathrm{TPI}$ & Canyons, DeeplyIncisedStraems & 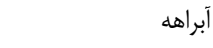 \\
\hline$-1<\mathrm{TPI}<1$, Slope $\leq \Delta^{\circ}$ & Plains & دشت \\
\hline$-1<\mathrm{TPI}<1$, Slope $\geq 0^{\circ}$ & Open Slopes & شيبهاى باز \\
\hline TPI>I & MountainTops, High Ridges & يالهاى مرتفع، قله كوه \\
\hline
\end{tabular}

Table 1. Land Form Classification based on TPI (75)
رطوبت خاك و تغييرات حاصل از فرسايش در خاك و عموماً

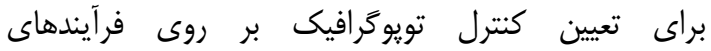

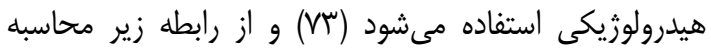

$\mathrm{TWI}=\ln \left(A_{s} \tan \beta\right)$

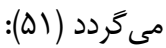

كه در رابطه فوق As مساحت ويزه حوزه آبخيز، م كَراديان شيب برحسب درجه و TWI شاخص رطوبت دوابت حوزه آبخيز مىباشد. اين شاخص نشاندهنده مدت زمان زمان تداوم انرزى جرئ ريان

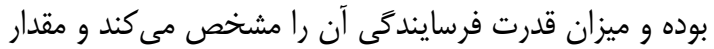

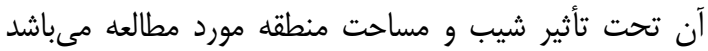

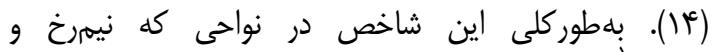

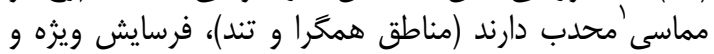

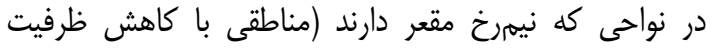
$\mathrm{SPI}=\mathrm{As} / \tan \beta$

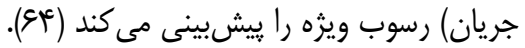

شاخص حمل رسوب

از جمله شاخصهاى هيله ريدرولوزيكى مورد استفاده در اين

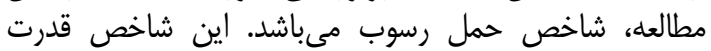

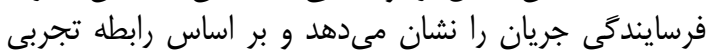
زير محاسبه مى زَردان

$\mathrm{STI}_{=}\left(\frac{A_{S}}{22.13}\right)^{0.6}\left(\frac{\operatorname{Sin} \beta}{0.0896}\right)^{1.3}$

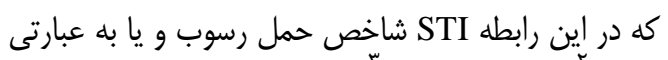

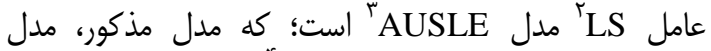

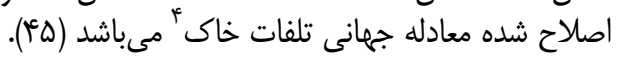

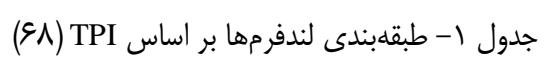
شاخص توان رودخانه
براى يارامتر p (احتمال وقوع يك يديده)، متغير وابسته كه ئه

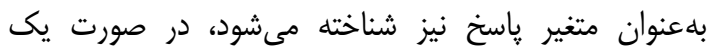

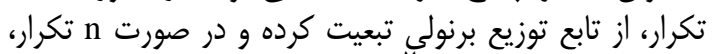

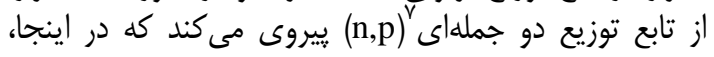

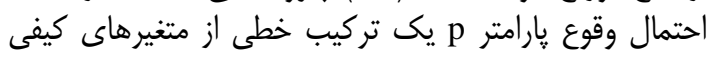

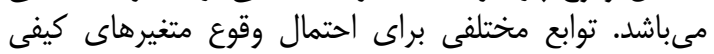

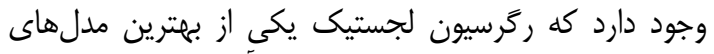

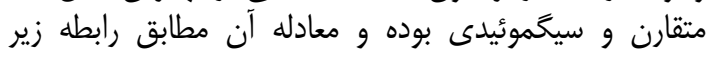
$\mathrm{p}=\frac{\exp (\beta X)}{1+\exp (\beta X)}$

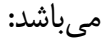

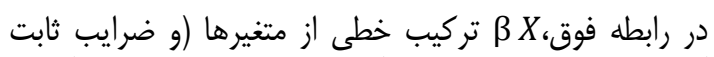

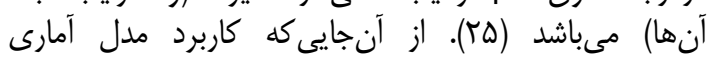

اجراى مدل رتر سيون لجستيكى

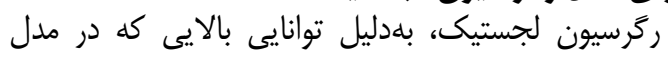

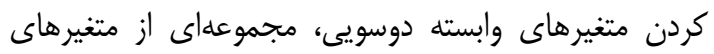

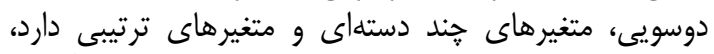

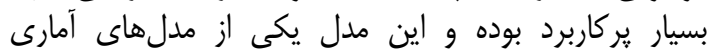

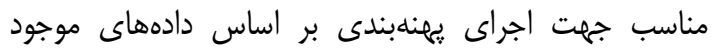

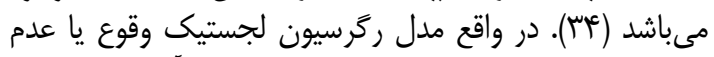

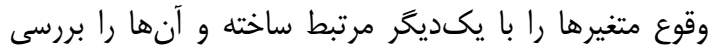

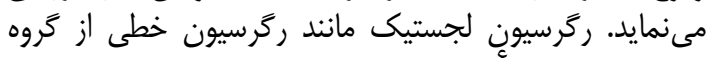

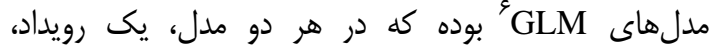

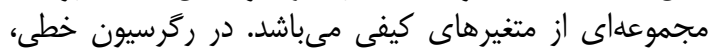

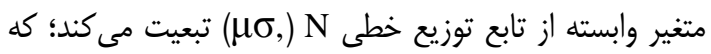

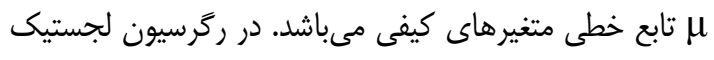

\begin{tabular}{lll}
\hline 1- Tangential & 2- Length and Slope & 3- Adapted Universal Soil Loss Equation \\
4- Universal SoilLoss Equation & 5- Land Form & 6-Generalized Linear Models \\
7- Binomial & &
\end{tabular}

7- Binomial 
يك واحد جابهجايى اعمال و انحراف طبقات جديد محاسبه

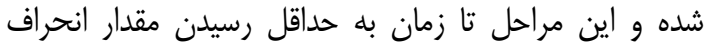

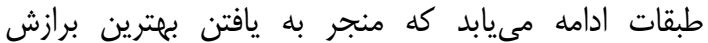

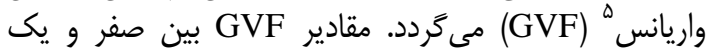
متغير بوده كه صفر بدترين و يك بهرديرين برادير براز مى باشد

\section{$\mathrm{GVF}=(\mathrm{SDAM}-\mathrm{SDCM}) / \mathrm{SDAM}$}

نتايج و بحث

يس از اجراى مدل لجستيك بر عوامل مؤثر بر وقوع

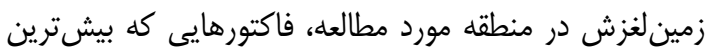

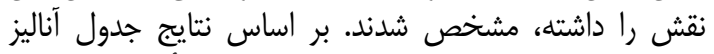

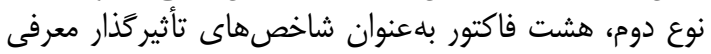

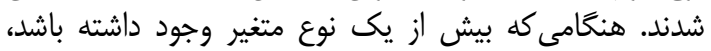

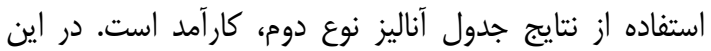

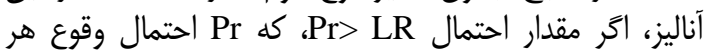

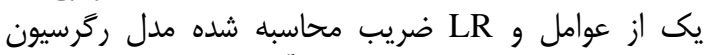

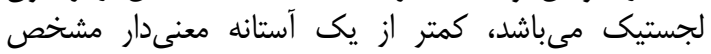

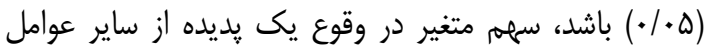

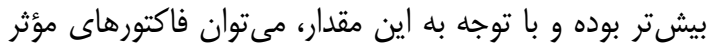

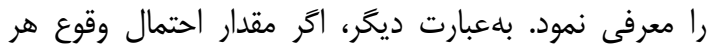
عامل از ضريب ركرسيون لجستيك بيشتر شود، مقدار سطح إنح

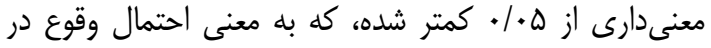

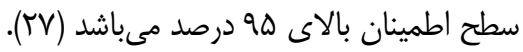

Table 2. Result of analyse type II

\begin{tabular}{|c|c|c|c|}
\hline $\operatorname{Pr}>L R$ & لايه مؤثر در زمينلغزش & $\operatorname{Pr}>\mathrm{LR}$ & لايه مؤثر در زمينلغزش \\
\hline 每 & شاخص SPI &.$/ .1$ & لايه خاك \\
\hline ( & شاخص يوشش گياهى &.$/ .4$ & لايه ارتفاع \\
\hline .11. & شاخص زبرى & r... & لايه زمين شناسى \\
\hline .11 .9 & لايه كاربرى اراضى & $\% t$ & شاخص TPI \\
\hline
\end{tabular}

ركرسيون لجستيك ايجاد ارتباط بين عوامل نإيايدار

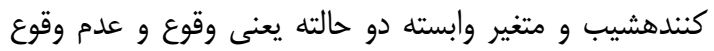

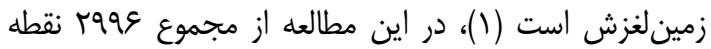

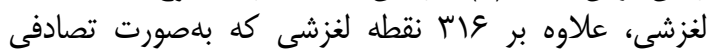

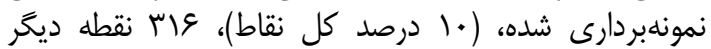

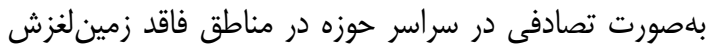

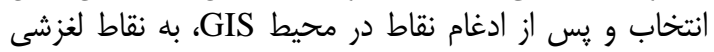
كد يك و به نقاط فاقد لغزش كد صناط دفر داده شد و و با استفاده از

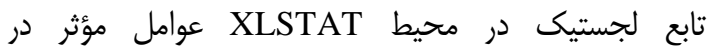

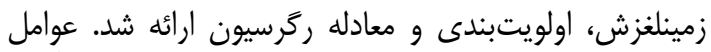

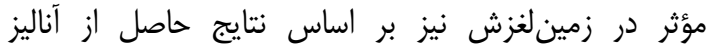

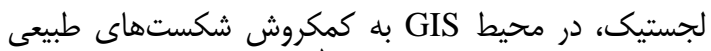

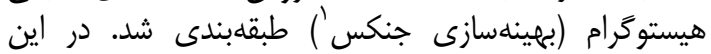

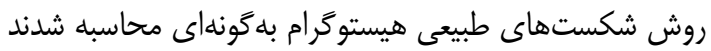

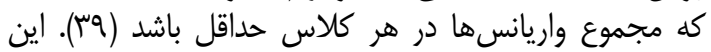

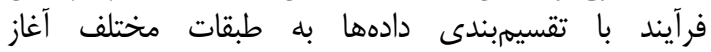

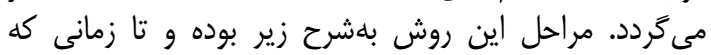

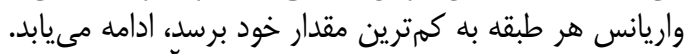

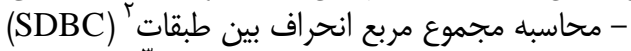

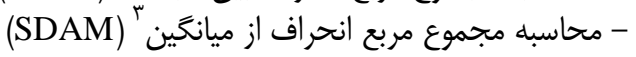
- محاسبه مجموع مربع انحراف از ميانئ مانكين طبقات

(SDCM)

گِس از محاسبه مقادير SDBC، از طبقهاى با بيشترين مقدار

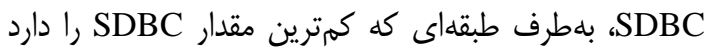

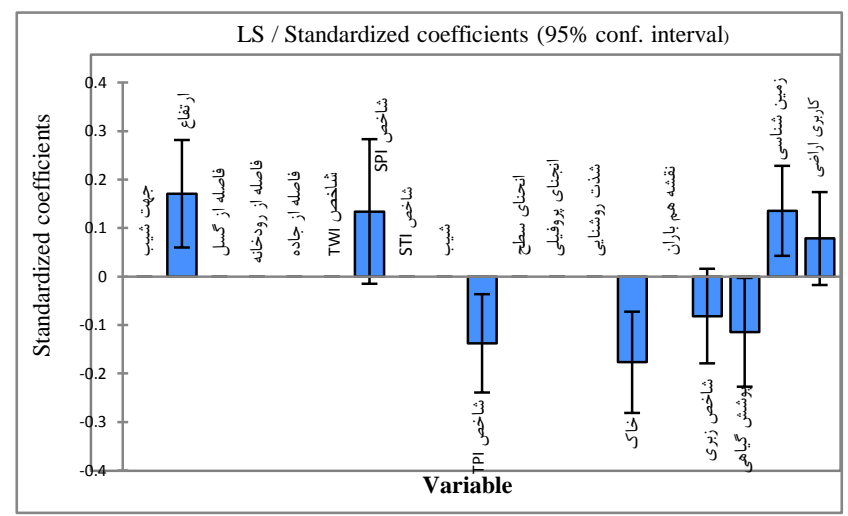

شكل r- ضرايب استاندارد شده در مدل ركرسيون لجستيك

Figure 2. Standardized coefficients in logistic regression model

متغيرها با يكديخر را نشان مىدهد، بهطورى كه مقادير مثبت

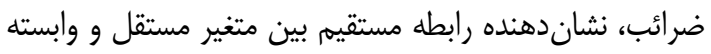

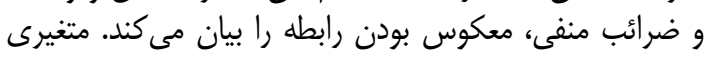

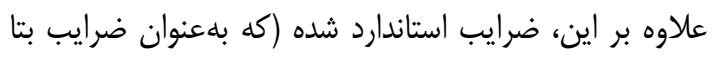

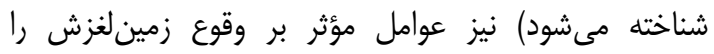

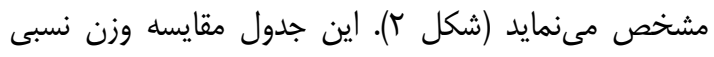


بالاى رس در مقايسه با ساير ذرات خاك، سبب پايدارى خاكدانه و ساختمان خاك شده (1)

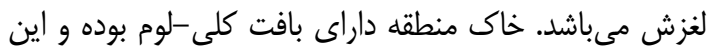

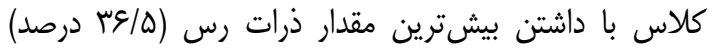

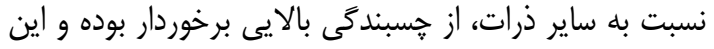

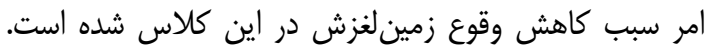

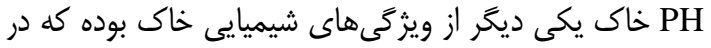

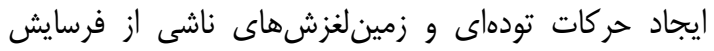

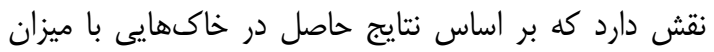

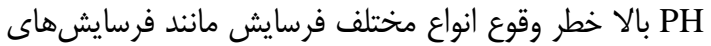

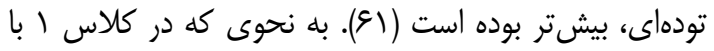
بيشترين لغزش ميزان PH، هـ هو و در كلاس بـ بـ با كمترين

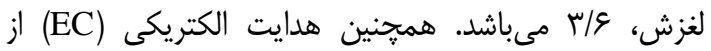

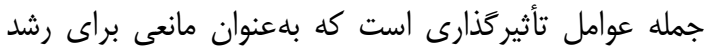

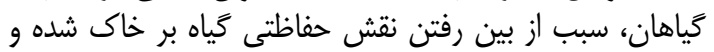

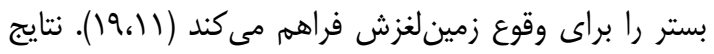

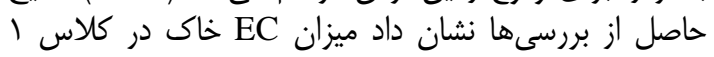

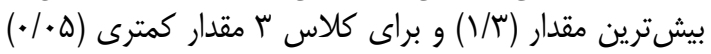

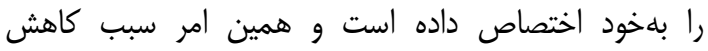

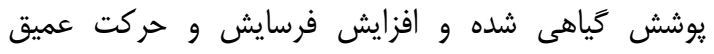
تودهاى خاك را در مناطق مذاه مذكور به همراه داش داشته است. از

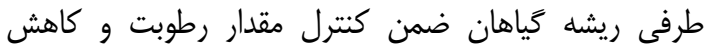

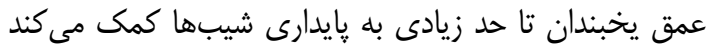

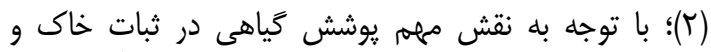

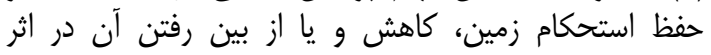

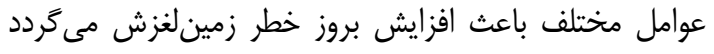

كه ضريب بالاترى نسبت به ساير متغيرها داشته باشد، از

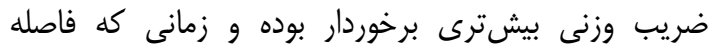
اطمينان در اطراف ضرايب استاندارد شده، مقادير صفر ران راب به

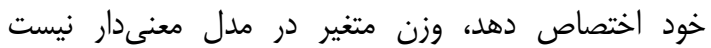

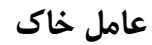

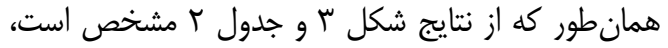

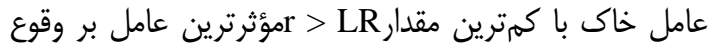

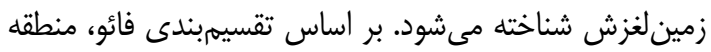

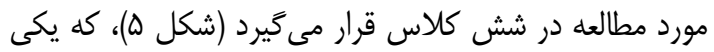

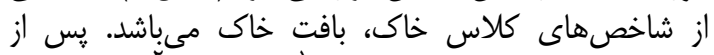

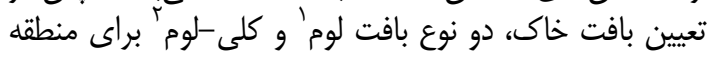

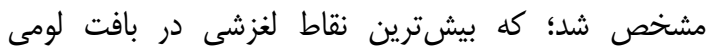

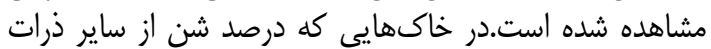

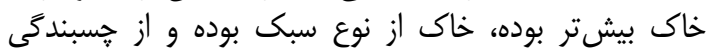

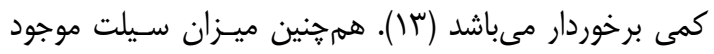

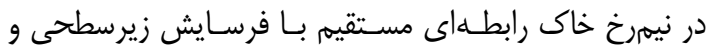

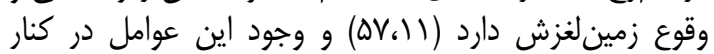

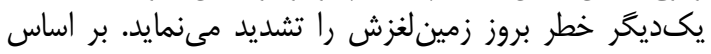

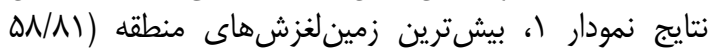

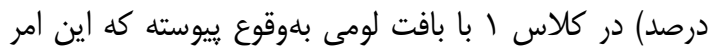

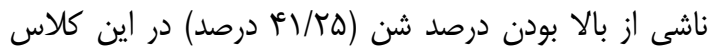

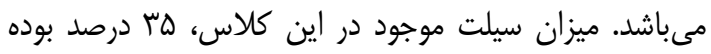

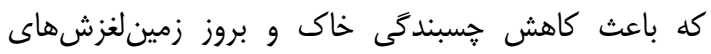

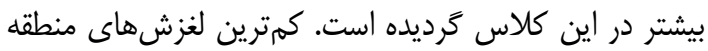
(1/ درصد) در كلاس ب مشاهده شده است. وجود مقادير لماري

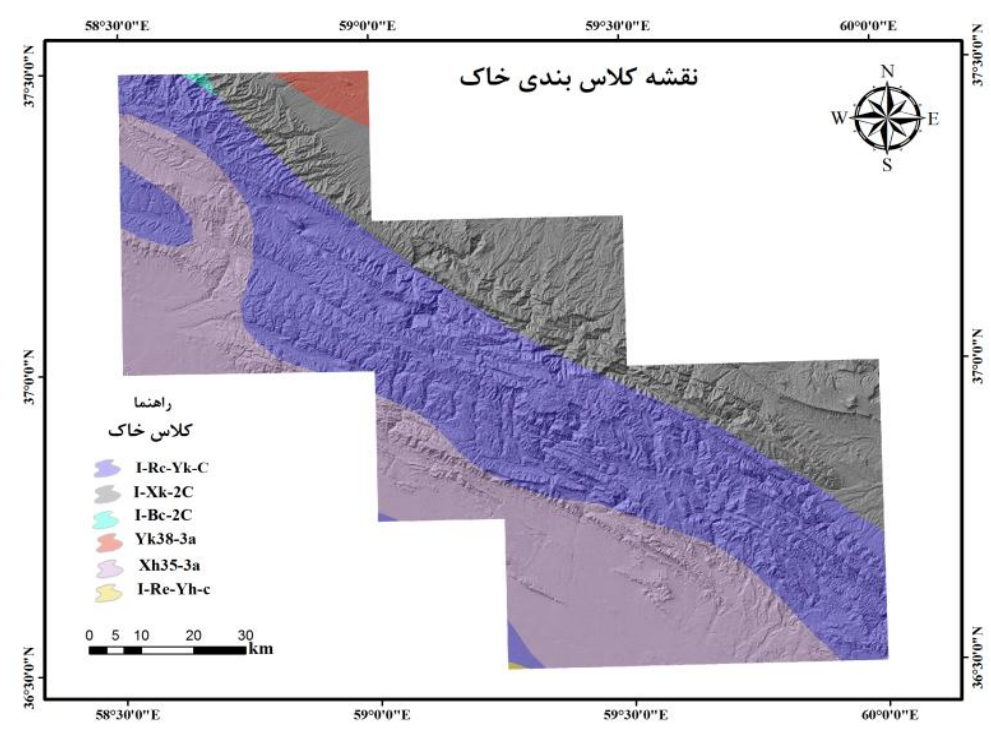

شكل س- نقشه طبقهبندى خاك منطقه مورد مطالعه

Figure 3. soil classes map of case study 


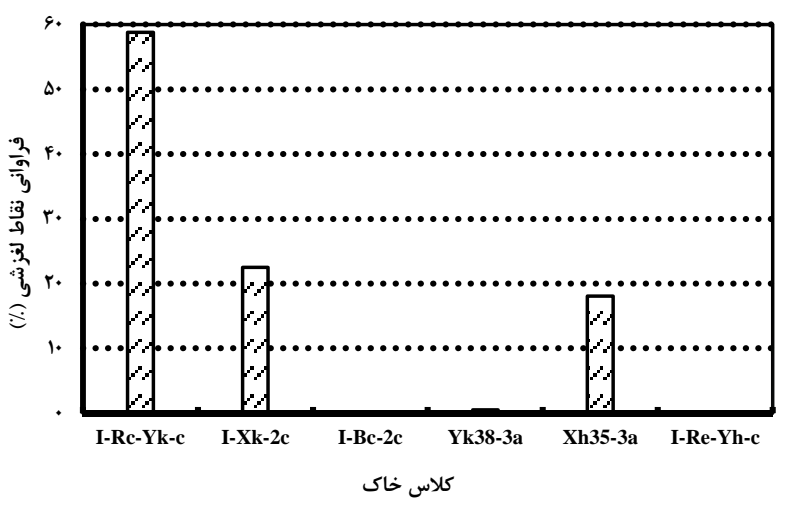

شكل f أ- نمودار درصد فراوانى نقاط لغزشى در هر كلاس خاك

Figure 4. Plot offrequency percentage of landslide points in soil classes

رابطه معكوس بين وقوع زمينلغزش و ارتفاعات بيشتر از

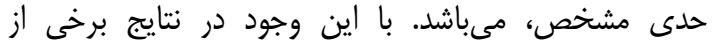

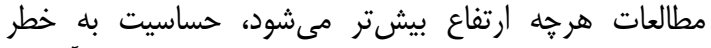

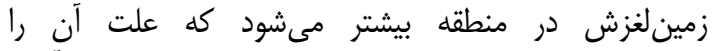

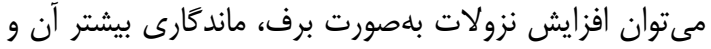

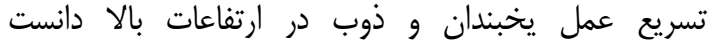

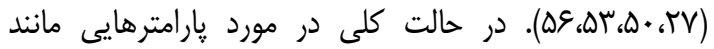

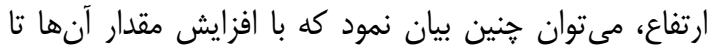

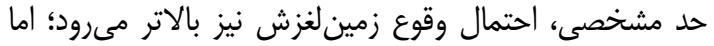

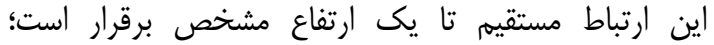

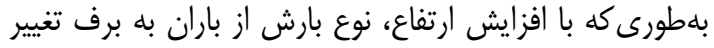

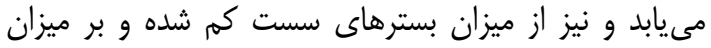

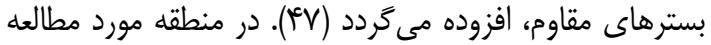

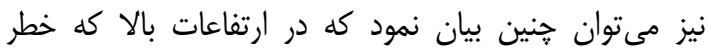

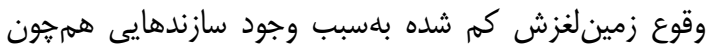

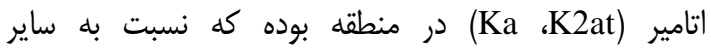

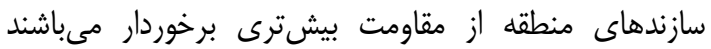

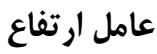

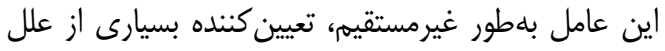

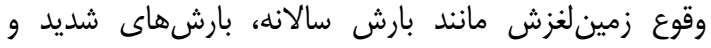

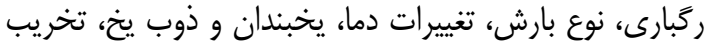

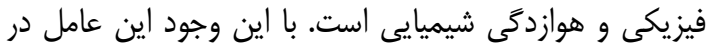

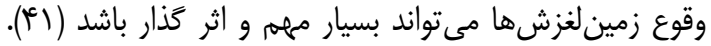

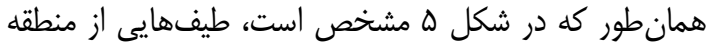

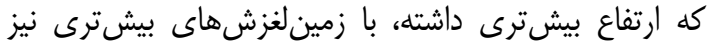

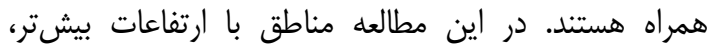

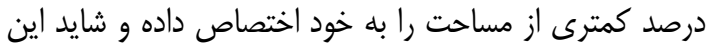

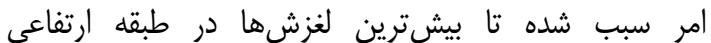

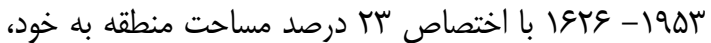

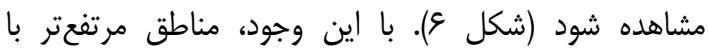

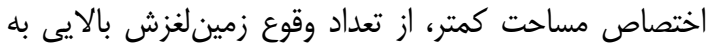

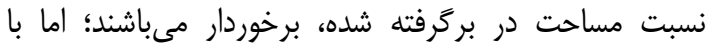

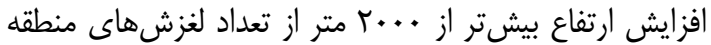
نسبت به ساير طبقات، كاسته مىشود و اين امر نشار لناندهنده

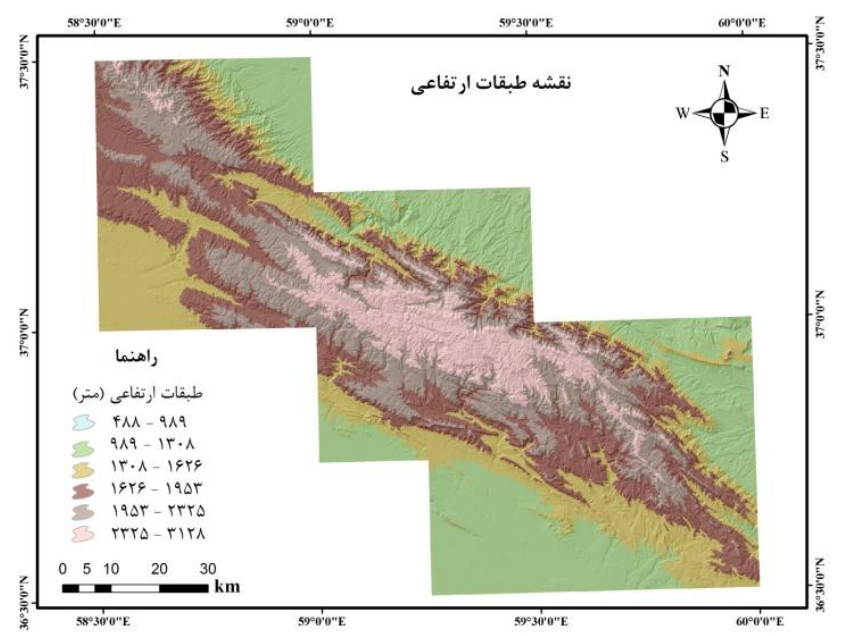

شكل ه- نقشه طبقات ارتفاعى موجود در منطقه مورد مطالعه

Figure 5. Elevation classes map of case study 


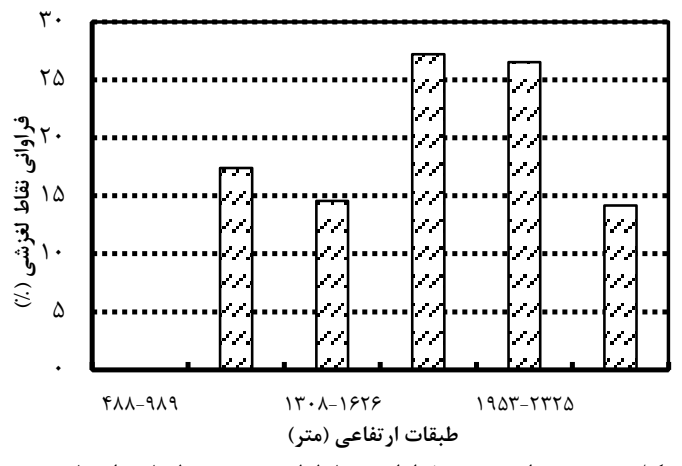

شكل צ- نمودار درصد فراوانى نقاط لغزشى در طبقاعتات ارتفاع

Figure 6. Plot of frequency percentage of landslide points in altitude classes

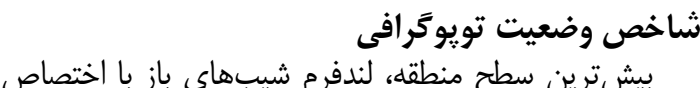
بيشترين سطح منطقها، لندفر

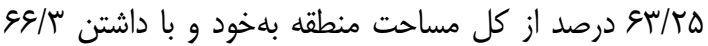

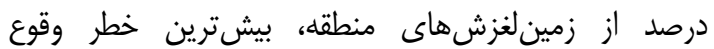

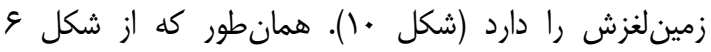

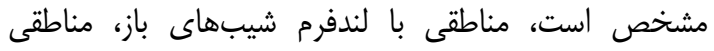

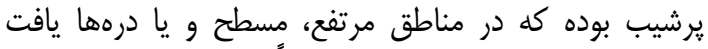

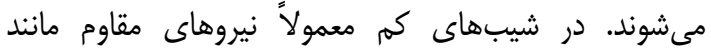

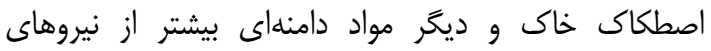

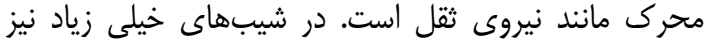

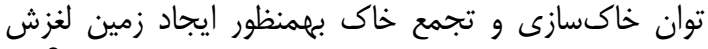

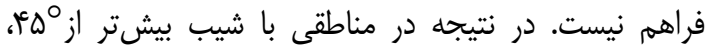

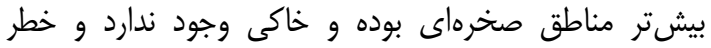

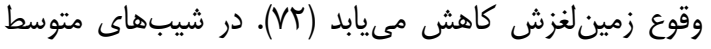

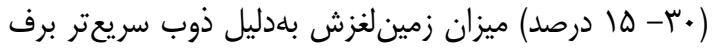

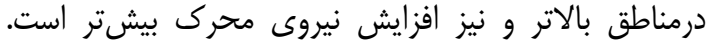

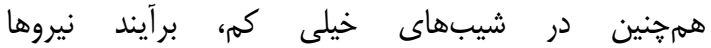

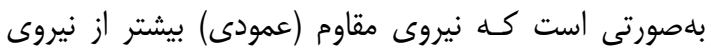

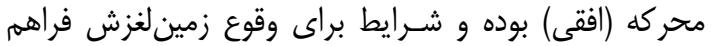

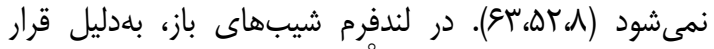

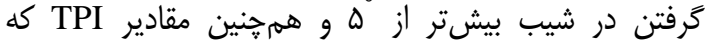

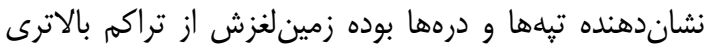

برخوردار بوده است (ه)مان.

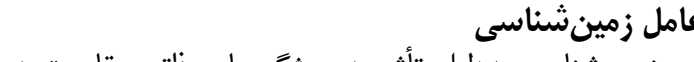

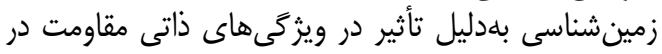

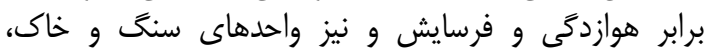

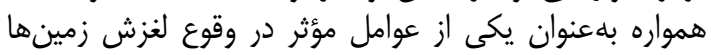

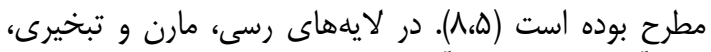

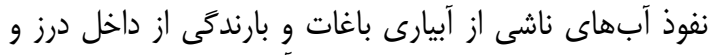

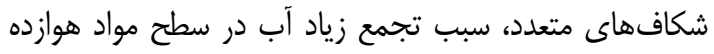

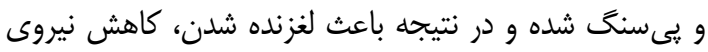

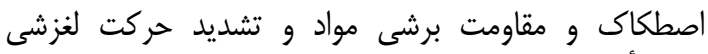

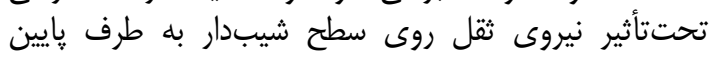

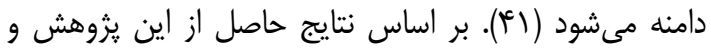

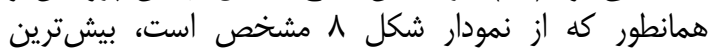
زمين لغزشها مربوط به واحد زمين شناسى Kt (سازند تيركان)

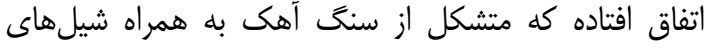

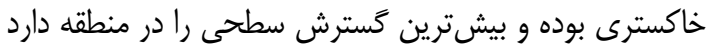

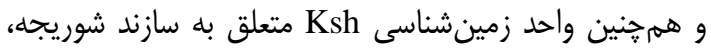

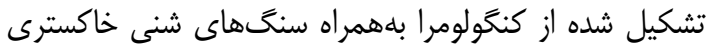

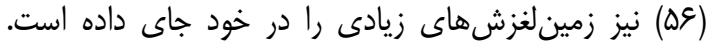

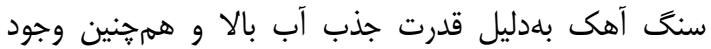

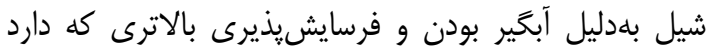

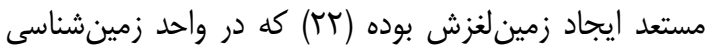

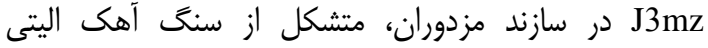
متوسط با شيلهاى فرعى سومين طبقه مستعد فرسايش الن الني

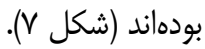

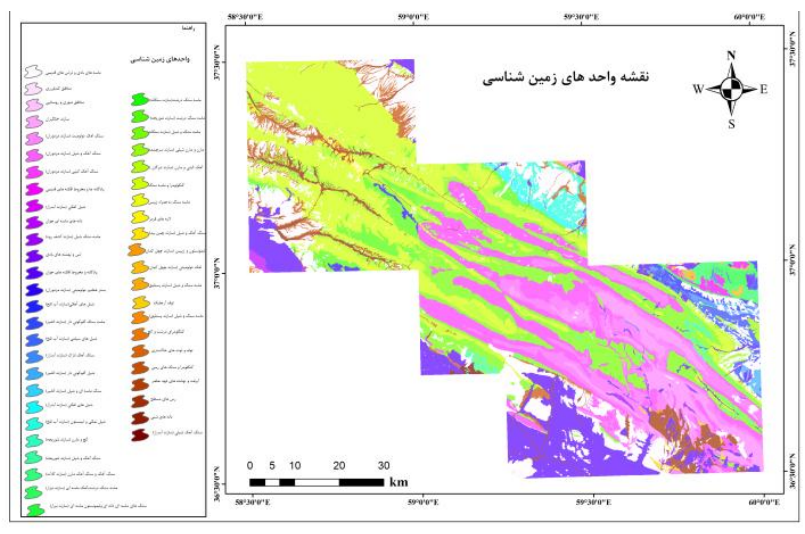

شكل V- نقشه واحدهاى زمينشناسى موجود در منطقه مورد مطالعه

Figure 7. Geology unit map of case study 


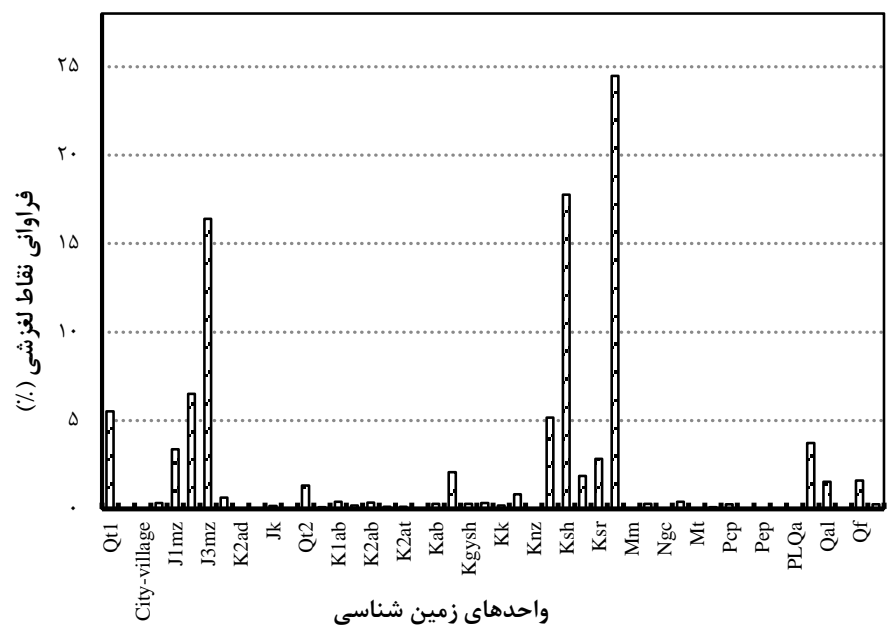

شكل 1- درصد فراوانى لغزش در واحدهاى زمينشناسى

Figure 8. Plot of frequency percentage of landslide points in Geology units

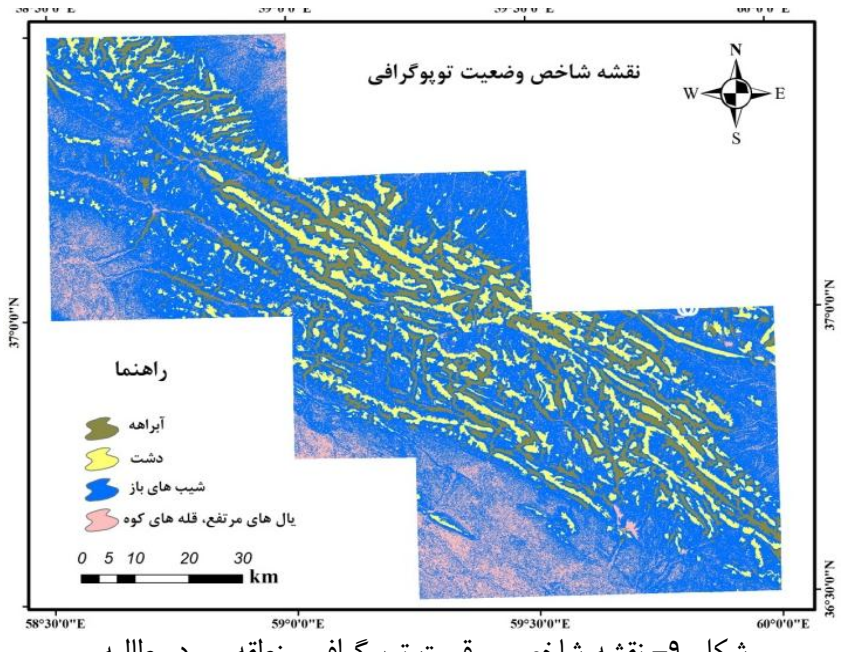

شكل 9- نقشه شاخص موقعيت تويوگر افى منطقه مورد مطالعه

Figure 9. Topographic Position Index map of case study

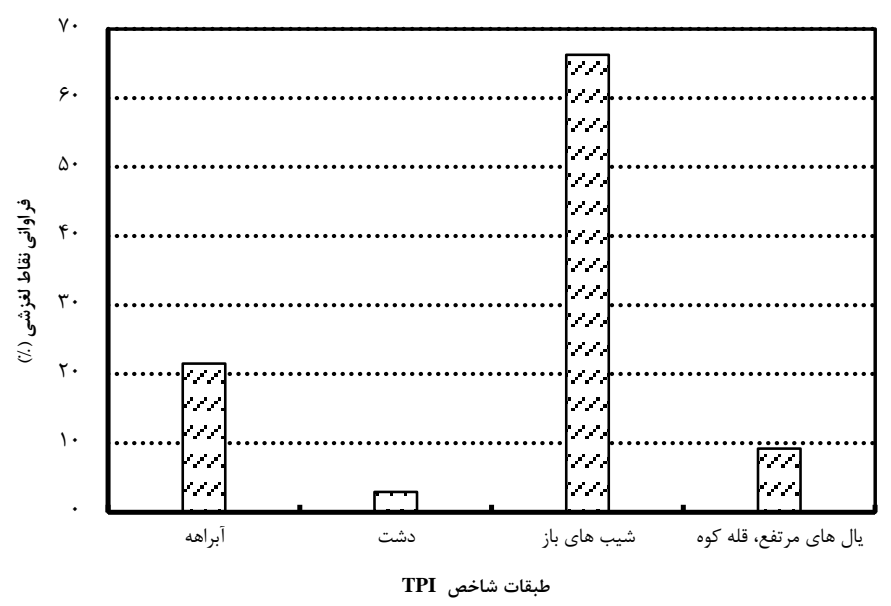

شكل • ا - نمودار درصد فراوانى لغزش در هر طبقه از شاخص TPI

Figure 10. Plot of frequency percentage of landslide points in TPI 


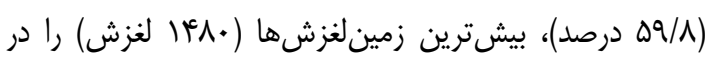

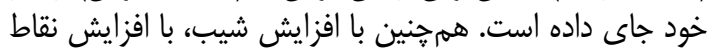

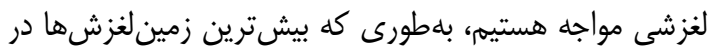

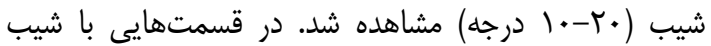

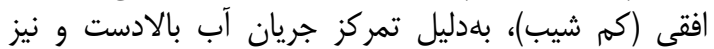

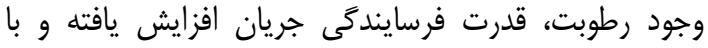

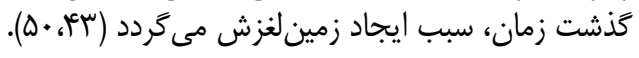

شاخص توان آبراهل

با افزايش شيب و مساحت حوزه، ميزان جريان آب بالا بالا

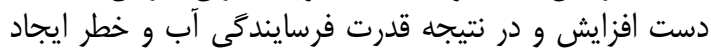

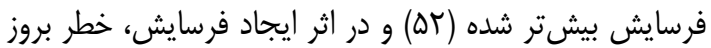

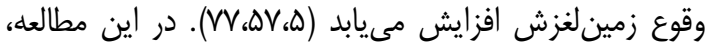

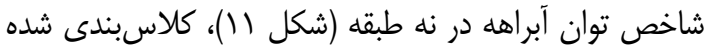

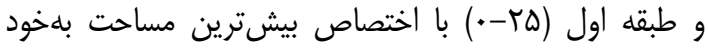

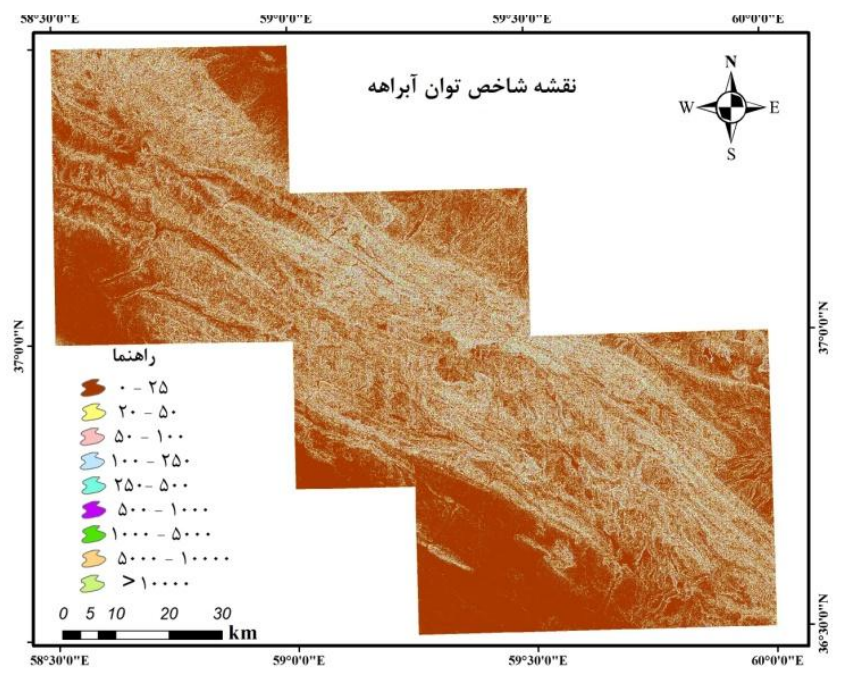

شكل II - نقشه طبقلهبندى شاخص SPI در منطقه مورد مطالعه

Figure 11.Stream Power Index map of case study

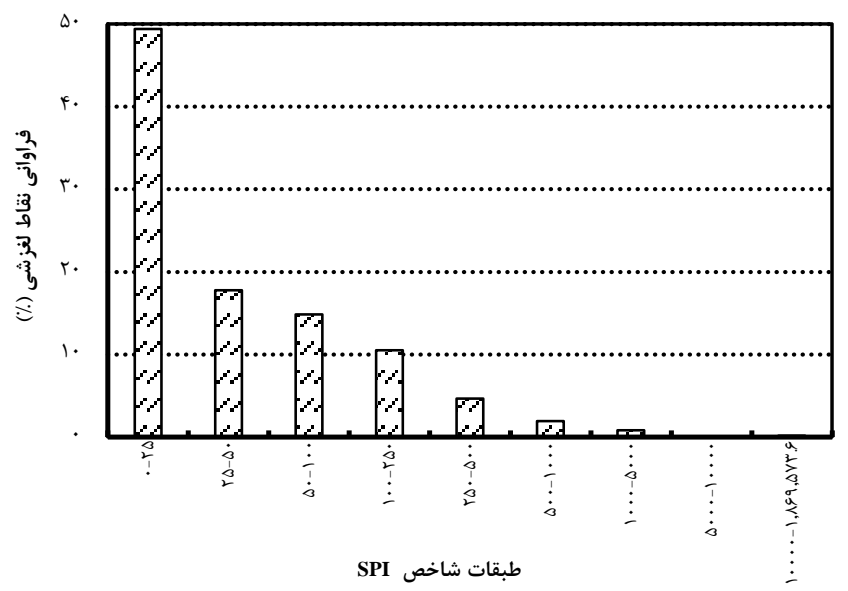

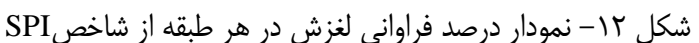

Figure 12. Plot of frequency percentage of landslide points in SPI classes

افتادهاست كه مناطقى با يوشش كياهى بسيار فقير مى باشد.

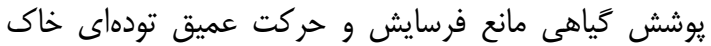

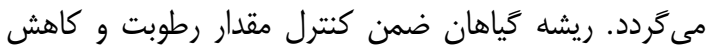

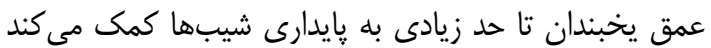

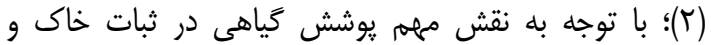

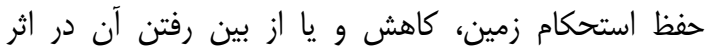
عوامل مختلف باعث افزايش بروز خطر زمين لغزش مي خَردد.
عامل شاخص يوشش كياهى

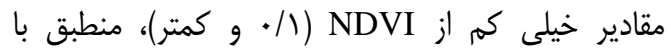

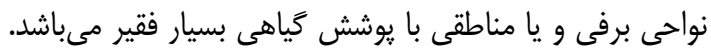

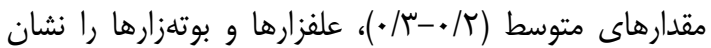

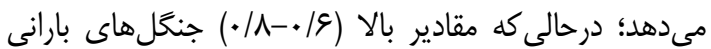

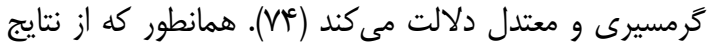

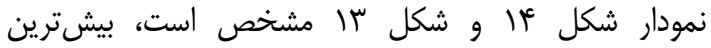

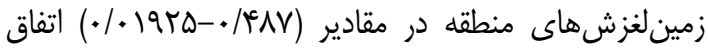




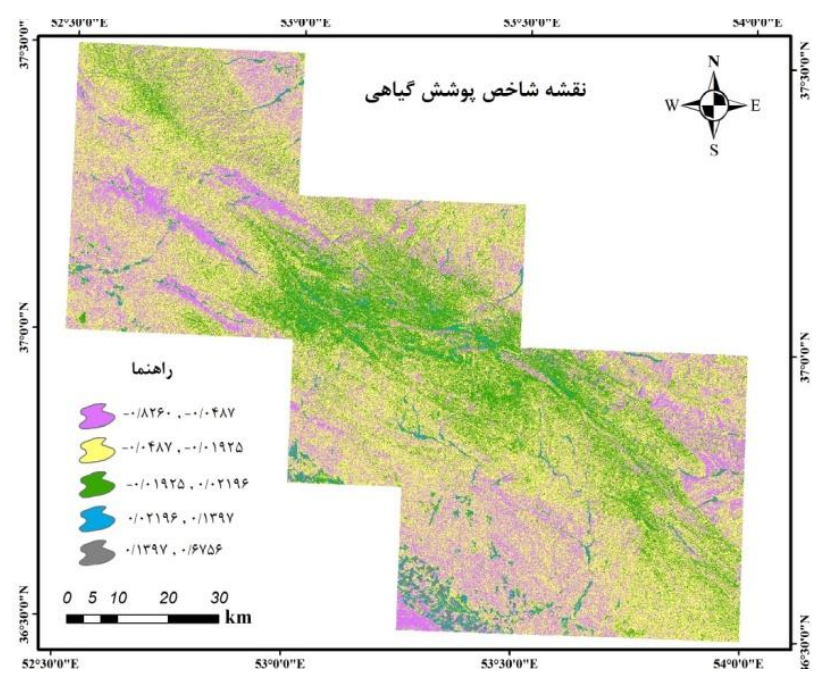

شكل سا - نقشه نرمال شده تفاوت يوشش كياهي منطقه مورد مطالعه Figure 13. NDVI Index map of case study

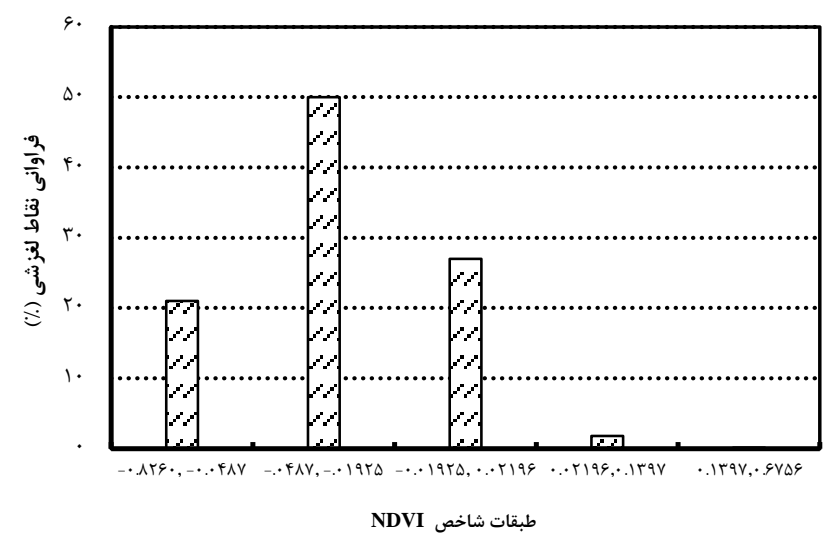

NDVI شكل عأ - نمودار درصد فراوانى لغزش در هر طبقه از شاخص

Figure 14. Plot of frequency percentage of landslide points in NDVI classes

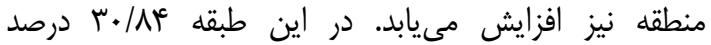

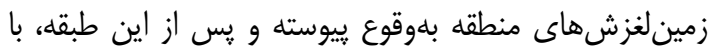

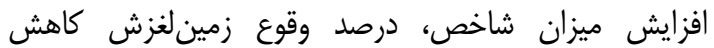

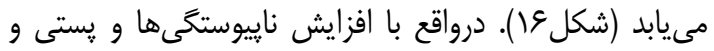

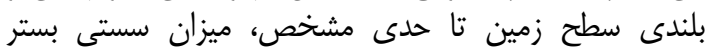
افزايش و درصد وقوع زمين لغزش افزايش مىيابد (ع) (شكل

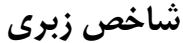

اين شاخص بهعن منوان فاصله عمودى بين سطح واقعى و

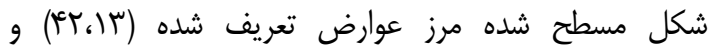

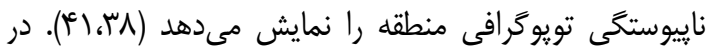

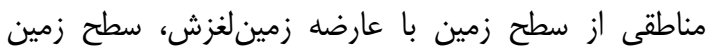

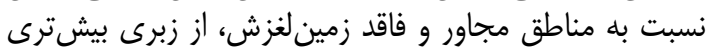

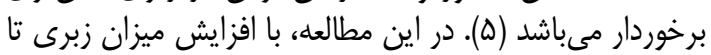

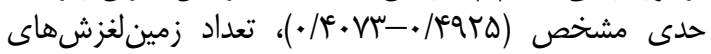




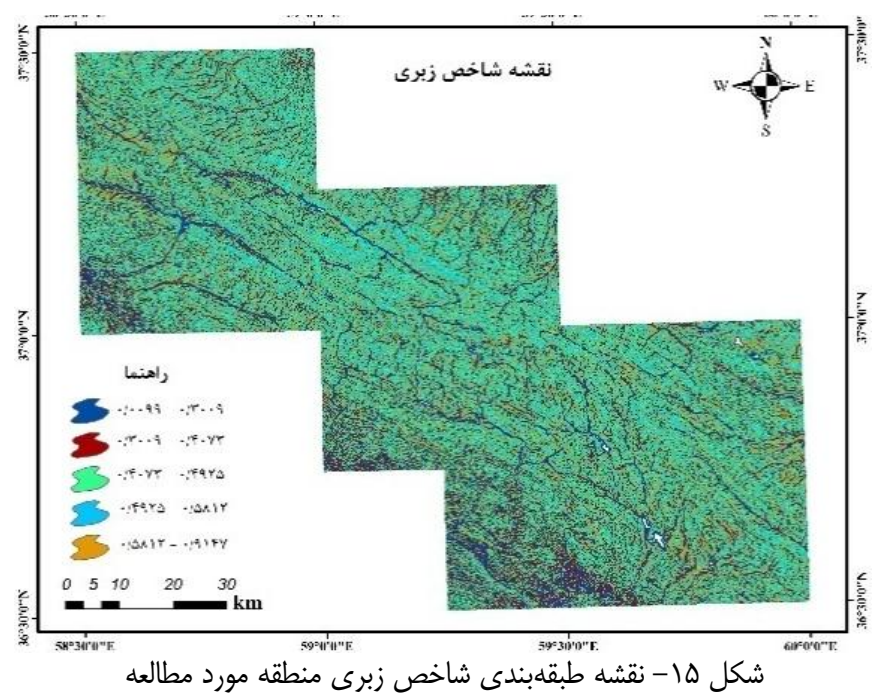

Figure 15. Roughness Index map of case study

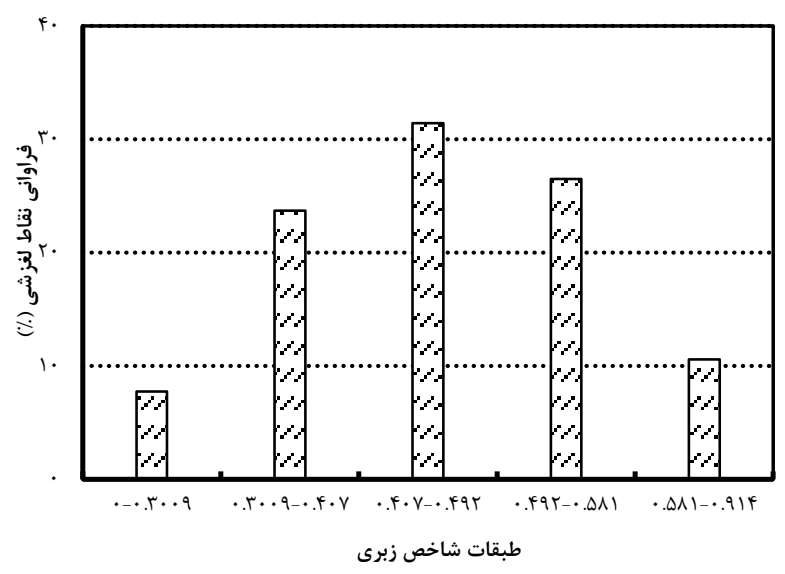

شكل عا - نمودار درصد فراوانى لغزش در طبقات شاخص زبرى

Figure 16. Plot of frequency percentage of landslide points in Roughness Index classes

است. در واقع بيشترين زمين لغزشهاى منطقه در كاربرى

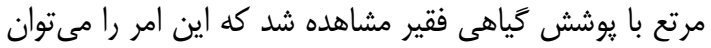

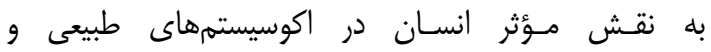

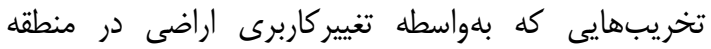

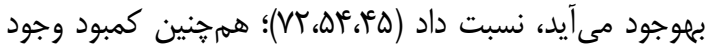

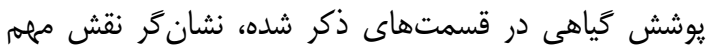

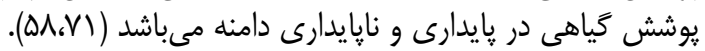

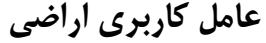

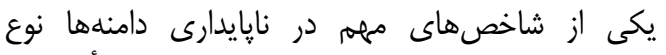

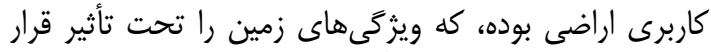

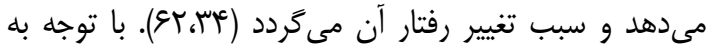

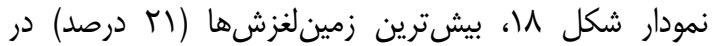

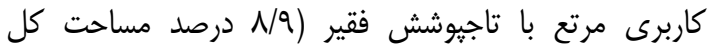

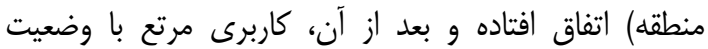

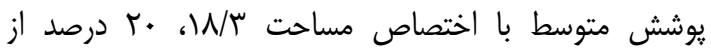
زمين لغزشهاى بلهوقوع ييوسته در منطقه را در خود جاى داد داده 


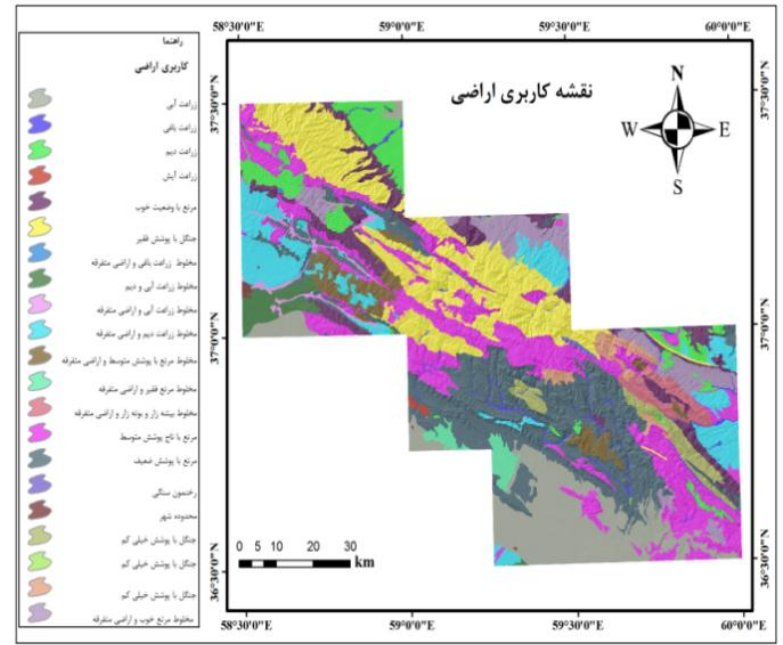

شكل Ve - نقشه كاربرى اراضى

Figure 17. Land use map

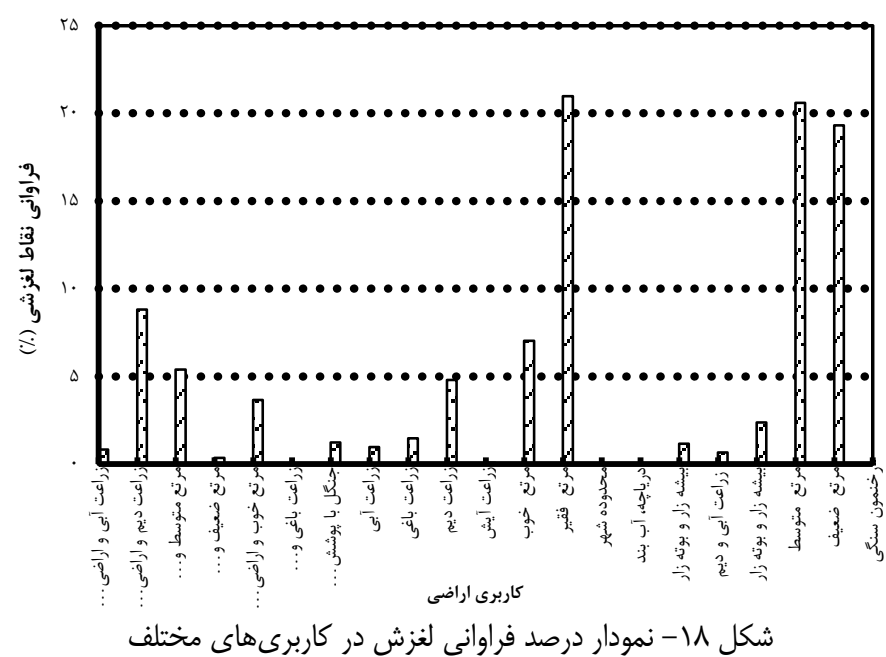

Figure 18. Plot of frequency percentage of landslide in different land uses

جريان نقش زيادى را در رخ داد زمينلغزش ايفا مىكنند.

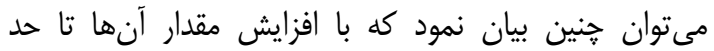

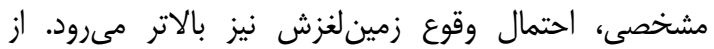

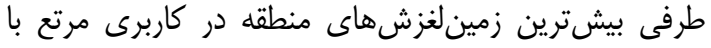

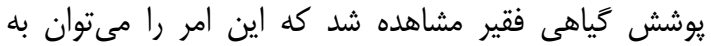

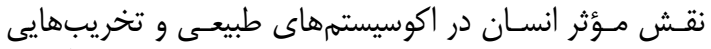

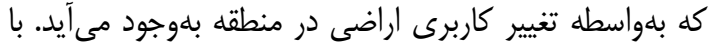

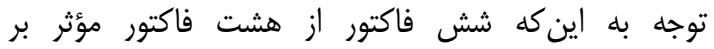

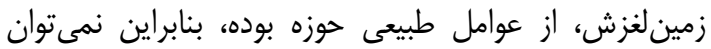

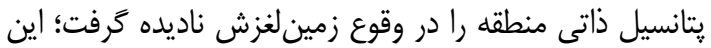

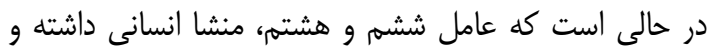

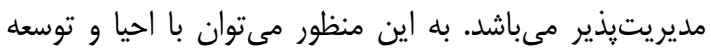

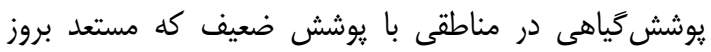

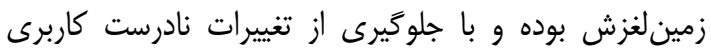

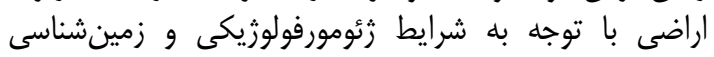
محل، خطر وقوع زمين لغزش را تا حد زيادى كاهش دادئ داد.
بلهور كلى مى توان خنين بيان نمود كه زمين لغزشها أنها

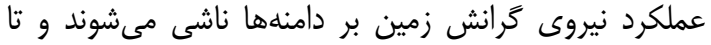

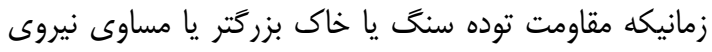

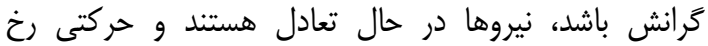

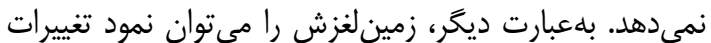

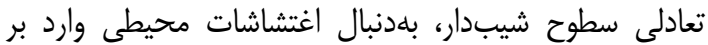

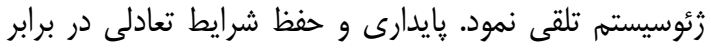

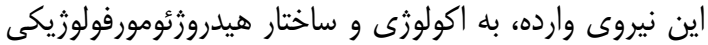
سطوح برمى

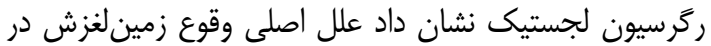

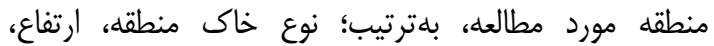

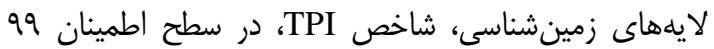
درصد كه با داشتن ضرائب استاندارد شده منفى شاخى و شاخص SPI

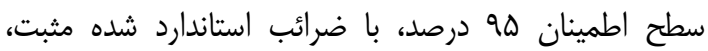

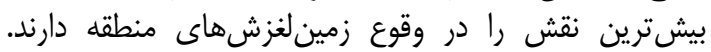

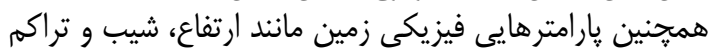




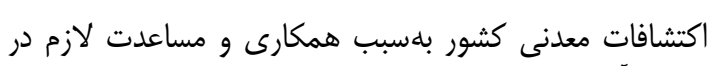

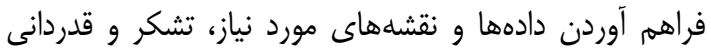
مى گردد.

$$
\begin{aligned}
& \text { قدردانى و تثكر }
\end{aligned}
$$

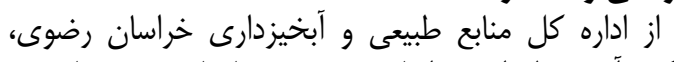

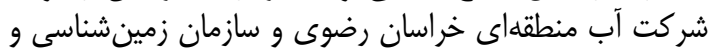

1. Abedini, M., B. Gasemyan and A.A. SHirzadi. 2015. Modelling the Hazard of Landslides by Using Statistical Method of Logistic Regression (Case study: Bijar of Kordestan). Journal of Geographi and development, 7(37): 85-102 (In Persian).

2. Basu, T. and S. Pal. 2018. Identification of landslide susceptibility zones in Gish River basin, West Bengal, India. Georisk. Journal of Assessment and Management of Risk for Engineered Systems and Geohazards, 12(1): 14-28.

3. Chen, Z. and J. Wang. 2007. Land slide hazard mapping using logistic regression model in Mskenzie Vally, Canada, Natural Hazards, 42: 75-89.

4. Choi, J., H.J. Oh, H.J. Lee, C. Lee and S. Lee. 2012. Combining landslide susceptibility maps obtained from frequency ratio, logistic regression, and artificial neural network models using ASTER images and GIS. Engineering Geology, 124: 12-23.

5. Convertino, M., A. Troccoli and F. Catani. 2013. Detecting fingerprints of landslide drivers: a MaxEnt model. Journal of Geophysical Research: Earth Surface, 118(3): 1367-1386.

6. Costanzo, D., E. Rotigliano, C. Irigaray Fernández, J.D. Jiménez-Perálvarez and J. Chacón Montero. 2012. Factors selection in landslide susceptibility modelling on large scale following the GIS matrix method: application to the river Beiro basin (Spain). Journal of Natural Hazards and Earth System Sciences, 12: $327-340$

7. Dahal, R.K., S. Hasegawa, A. Nonomura, M. Yamanaka, S. Dhakal and P. Paudyal. 2008. Predictive modelling of rainfall-induced landslide hazard in the Lesser Himalaya of Nepal based on weights-ofevidence. Journal of Geomorphology, 102: 496-510.

8. Darvishzadeh, A. 2005. Geology of Iran. Amir Kabir press, Tehran, Iran. 901 pp (In Persian).

9. Deng, X., L. Li and Y. Tan. 2017. Validation of Spatial Prediction Models for Landslide Susceptibility Mapping by Considering Structural Similarity. ISPRS International Journal of Geo-Information,6: 1-16.

10. Ebrahimi, M., M. Habibolelahiyan and A. Amir Ahmadi. 2015. Investigating the effect of road construction on surface landslides occurrence using slopes stability model, (Case study: Kalat Watershed). Journal of Geographical Planing of Space Quarterly, 59(15): 149-162 (In Persian).

11. Emami, S.N., A. Jalaliyan and A. Khosravi. 2016. The role of physical and chemical properties of soil in the occurrence of landslide, (Case study: Afsar Abad Chaharmahal va Bakhtiari). Journal of Watershed Management Research, 7(13): 182-192 (In Persian).

12. Ezati. M., M. AghAtabai, M. Raghimi and Sh. Shataee. 2012. Analysis of Some Morphotectonic Indices of Shirinrud Subcatchment, (Case study: Central Kopeh Dagh). Journal of Geographic Planning of Space Quarterly, 2(6): 1-16 (In Persian).

13. FazeliSHahroodi, E. and S.A. Hosseyni. 2013. Investigating the Factors of Occurrences of forest andslide. $8^{\text {th }}$ Iranian Engineering and Environmental Geology Congress, Mashhad, Iran, 1-9 (In Persian).

14. Florinsky, I. 2016. Digital terrain analysis in soil science and geology. Academic Press, 506 pp.

15. Fooruzanfar, M., GH.Vahabzadeh and H.R. Pourghasemi. 2014. Landslide Susceptibility Mapping Using Fusion Models of Frequency Ratio (FR) and Analytical Hierarchy Process (AHP) (Case study: Talar basin). Journal of Environmental Erosion Research, 3(4): 17-29 (In Persian).

16. Garfi, G., D.E. Bruno, D. Calcaterra and M. Parise. 2007. Fan morph dynamics and slope instability in the Mucone River basin (Sila Massif, southern Italy): significance of weathering and role of land use changes. Catena, 69: 181-196.

17. Ghafouri, M. and A.R. Ashouri. 1997. Landslide as one of the natural disasters in northern Khorasan. Journal of Geographic Research, 445: 90-101.

18. Ghanavati, E.A. 2011. Land slide zoning using hierarchical analysis method (Case study: Jajrood basin). Journal of Applied Geosciences Research, 8(20): 51-68 (In Persian).

19. Gharehshaeikhloo, A.H., M.R. Vahabi and H.R. Karimzadeh. 2010. Comparison of soil characteristics of land withlwithout vegetation (Case study: Dagh-e-SorkhArdestan catchment). Journal of Science and Technology of Agriculture and Natural Resources, 14(53): 89-97 (In Persian).

20. Ghavimipanah, M.H., A.A. Khaledi Darvishan and M.R. Ghavimipanah. 2017. Verification Methods of Analytical Hierarchy Process (AHP) and Multivariate Regression (MR) in Landslide Zoning (Case Study: Valiasr Watershed in Ardabil Province). Journal of Ecohydrology, 4(3): 775-789 (In Persian).

21. Goetz, J.N., R.H. Guthrie and A. Brenning. 2011. Integrating physical and empirical landslide susceptibility models using generalized additive models.Journal of Geomorphology, 129 (3-4): 376-386.

22. Gorum, T., B. Gonencgil, C. Gokceoglu and H.A. Nefeslioglu. 2008. Implementation of reconstructed geomorphologic units in landslide susceptibility mapping: the Melen Gorge (NW Turkey). Natural Hazards, 46: 323-351.

23. Hall, F.G., J.R. Townshend and E.T. Engman. 1995. Status of remote sensing algorithms for estimation of land surface state parameters. Journal of Remote Sensing of Environment, 51(1): 138-156. 
24. Hemati, F. and S.A. Hejazi. 2017. Landslide hazard zonation using logistic regression statistical method (Case study: Lavasanat Watershed). Applied Researches in Geographical Seiences, 17(45): 7-24 (In Persian).

25. Hosmer, D.W. and S. Lemeshow. 2000. Applied Logistic Regression, Second Edition. John Wiley and Sons, New York. Doi: 10.1002/0471722146.

26. Hosseinzadeh, M.M., M.R. Servati, A. Mansoori, B. Mirbagheri and S. Khazri. 2009. Zoning the risk of massmovement occurrences using logestic regression model, (Case study:in vicinity of Sanandaj-Dehgolan road). Journal of Geology, 3(11): 27-37 (In Persian).

27. Ilanloo, M., E. Moghimi, M.R. Servati and M. GhahroodiTali. 2010. Zoning the Risk of Mass Moves Using Fuzzy Logic Method (Case Study: Sierra Watershed). Journal of Georraphical Landscape, 5(11): 12-26 (In Persian).

28. Ildormi, A.R., H. Nori, M. Naderi, A. Aghbeigi and H. Zeinivand. 2015. Land use Change Prediction using Markov Chain and CA Markov Model, (Case Study: Gareen Watershed). Journal of Watershed Management Research, 8(16): 232-240 (In Persian).

29. Jenks, G.F. 1967. The data model concept in statistical mapping. International yearbook of cartography,7: 186-190.

30. Jenness, J. 2006. Topographic Position Index extension for ArcView 3. x, v. 1.3 a. Jenness Enterprises.[online: www. jennessent. com/arcview/tpi. htm].

31. Kalarestaghi, A.A., M. Habibnejad and H. Ahmadi. 2007. Study of landslide occurrence in relation to land use change and road construction, (Case study: Tajan watershed, Sari). Journal of Geographic research, 1849: 81-91.

32. KarimiSangchini, E., M. Ownegh and A. Sadoddin. 2016. Landslide Hazard Management for Two Normal and Critical Scenarios in the Chehel-Chay Watershed, Golestan Province. Journal of Watershed Management Research, 7(13): 173-181.

33. Lee, C.T., C.C. Huang, J.F. Lee, K.L. Pan, M.L. Lin and J.J. Dong. 2008. Statistical approach to earthquake-induced landslide susceptibility. Journal of Engineering Geology, 100: 43-58.

34. Lee, S.A. 2004. Verification of Spatial Logistic Regression for Landslide Susceptibility Analysis: A Case Study of Korea. Journal of Geomorphology, 44: 15-18.

35. Lillesand, T., R.W. Kiefer and J. Chipman. 2014. Remote sensing and image interpretation. John Wiley \& Sons, Hoboken.

36. Lindsay, J.B. 2005. The terrain analysis system: A tool for hydro-geomorphic applications. Hydrological Processes, 19(5): 1123-1130.

37. Liu, H.Q. and A. Huete. 1995. A feedback based modification of the NDVI to minimize canopy background and atmospheric noise. IEEE Transactions on Geoscience and Remote Sensing, 33: 457-465.

38. Maleki, A., H. Marabi and H. Rahimi. 2016. Evaluation of Topographic Position Index (TPI), (Case Study: Zone Sanandaj-Sirjan and Broken Zagros). Journal of Quantitative Geomorphology Research, 5(1): 129141 (In Persian).

39. Memarian, H., S.K. Balasundram, J.B. Talib, C.T.B. Sung, A.M. Sood and K. Abbaspour. 2012. Validation of CA-Markov for simulation of land use and cover change in the Langat Basin, Malaysia. Journal of Geographic Information System, 4(6): 542-554.

40. Memarian, H., M. Tajbakhsh, A. Safdari and E. Akhondi. 2006. Statistical Landslide Risk Zonation on the Shourijeh Formation in GIS Framework, (Case study: Estarkhy Watershed in the Northeast of Iran).Geomatics Conference, Tehran, 1-11.

41. Moghimi, A., S.K. Alavipanah and T. Jafari. 2008. Evaluation and Zonation of Effective Factors on Landslide Occurrence of Aladagh Northern Slopes (Case study: Chenaran drainage basin in North Khorasan Province) .Quarterly. Journal of Geographical Research, 64: 53-75 (In Persian).

42. Mohammadi, M., H.R. Moradi, S. Feiznia and H.R. Pourghasemi. 2009. Landslide hazard mapping using density area and AHP models. Journal of Watershed Engineering and Management, 1(4): 248-258.

43. Mondal, S. and S. Mandal. 2017. RS\&GIS-based landslide susceptibility mapping of the Balason River basin, Darjeeling Himalaya, using logistic regression (LR) model. Journal of Georisk: Assessment and Management of Risk for Engineered Systems and Geohazards, 1-16.

44. Moore, I.D., P.E. Gessler, G.A. Nielsen and G.A. Peterson. 1993. Soil attribute prediction using terrain analysis.Soil Science Society of America Journal, 57: 443-452.

45. Moore, I.D., R.B. Graysonand A.R. Ladson. 1991. Digital terrain modelling: a review of hydrological, geomorphological, and biological applications. Journal of Hydrological Processes, 5: 3-30.

46. Moradi, H.R., M. Mohamadi and H.R. Poorghasemi. 2014. Domain moves (mass movements) with emphasis on quantitative methods of landslide analysis. Samtperss, 224 pp (In Persian).

47. Moradi, H.R., H.R. Pourghasemi, M. Mohammadi and M.R. Mahdifar. 2011. Landslide hazard zoning using Gamma fuzzy operator (Case study: Haraz watershed). Journal of Natural science, 7(4): 129-142 (In Persian).

48. Nachtergaele, F., H. van Velthuizen, L. Verelst, N. Batjes, K. Dijkshoorn, V. van Engelen, G. Fischer, A. Jones, L. Montanarella, M. Petri, S. Prieler, E. Teixeira, D. Wiberg and X. Shi. 2009. Harmonized world soil database. Wageningen: ISRIC, 1-43.

49. Negahban, S. and M. Mokarami. 2015. Landform classification using Topographic Position Index (TPI) (Case study: Hakan watershed, Jahrom city). Journal of Environmental Erosion Research, 5(17): 75-89 (In Persian). 
199 يُزوهشنامه مديريت حوزه آبخيز سال دهم/ شماره 19/ بهار و تابستان حوسا .

50. Nefeslioglu, H.A., T.Y. Duman and S. Durmaz. 2008. Landslide susceptibility mapping for a part of tectonic Kelkit Valley (Eastern Black Sea region of Turkey).Geomorphology, 94(3-4): 401-418.

51. Ocakoglu, F., C .Gokceoglu and M. Ercanoglu. 2002. Dynamics of a complex mass movement triggered by heavy rainfall: a case study from NW Turkey. Journal of Geomorphology, 42(3): 329-341.

52. Ohlamcher, G.C. and J.C. Davis. 2003. Using multiple logistic regression and GIS technology to predict landslide hazard in northeast Kansas USA. Journal of Engineering Geology, 69: 331-343.

53. Pachauri, A.K. and M. Pant. 1992. Landslide hazard mapping based on geological attributes. Journal of Engineering Geology, 32(1-2): 81-100.

54. Pourghasemi, H.R., M. Mohammady and B. Pradhan. 2012. Landslide susceptibility mapping using index of entropy and conditional probability models in GIS: Safarood Basin, Iran. Catena, 97: 71-84.

55. Pourghasemi, H.R., B. Pradhan and C. Gokceoglu. 2012. Application of fuzzy logic and analytical hierarchy process (AHP) to landslide susceptibility mapping at Haraz watershed, Iran. Natural hazards, 63: 965-996.

56. Pourghasemi, H.R., H.R. Moradi and S.M. Fatemi Oghda. 2015. Prioritizing the factors affecting landslide occurrence and zoning its sensitivity using Shannon Entropy Index, (Case Study: North of Tehran). Journal of Science and Technology of Agriculture and Natural Resources, 18(70): 181-192 (In Persian).

57. Raffatniya, N.A., M.K. Kaviyanpoor and T. Ahmadi. 2011. The study of landslide in Glendrood forest, (Case study: Distriact 3 from catchment area 48). Journal of Science and Technology of Agriculture and Natural Resources, 6(1): 53-63 (In Persian).

58. Rasai, A., Kh. Khosravi, M. Habibnejad Roshan, A. Heidari and A. Mashayekh Khan. 2015. Lnadslide Hazard Zonation using Multivariate Regression in GIS Environment (Case Study: Aghmashhad Watershed, Mazandaran). Juornal of Watershed Management Research, 6(12): 205-215 (In Persian).

59. Różycka, M., P. Migoń and A. Michniewicz. 2017. Topographic Wetness Index and Terrain Ruggedness Index in geomorphic characterisation of landslide terrains, on examples from the Sudetes, SW Poland. Zeitschriftfür Geomorphologie, Supplementary Issues, 61(2): 61-80.

60. Saldivar-Sali, A. and H.H. Einstein. 2007. A landslide risk rating system for Baguio, Philippines. Journal of Engineering Geology, 91: 85-99.

61. Salmasi, R. and A. Ahmadi. 2012. Relationship between Some Properties of Marls and Different Types of Erosions in Talkhe-rood Watershed, Journal of Geography and sustainability of Environment, 2(3): 11-23 (In Persian).

62. Seyed-Emami, K. and A.A. Aryai. 1981. Ammoniten aus dem unteren Cenoman von Nordostiran (KoppehDagh). Mitteilungen der Bayerischen Staatssammlung für Paläontologie und historische Geologie, 21(2): 23-39.

63. Shahabi, H., S. Khezri, B.B. Ahmad and M. Hashim. 2014. Landslide susceptibility mapping at central Zab basin, Iran: a comparison between analytical hierarchy process, frequency ratio and logistic regression models.Catena, 115: 55-70.

64. Sharma, B., N. Sharma and N. Sharma. 2010. Simulation of different SPI Models. International Journal of Computer Applicationz, 10: 1-6.

65. Shirani, K. and A.R. Arab Ameri. 2015. Landslide Hazard Zonation Using Logistic Regression Method (Case Study: Dez-e-Oulia Basin). Journal of Science and Technology of Agriculture and Natural Resources, 19(72): 321-334 (In Persian).

66. Sörensen, R., U. Zinko and J. Seibert. 2006. on the calculation of the topographic wetness index. Evaluation of different methods based on field observations. Journal of Hydrology and Earth System Sciences Discussions, 10: 101-112.

67. Weier, J. and D. Herring. 2005. Measuring Vegetation (NDVI and EVI). Earth Observatory Library of NASA. /http.Earthobservatory. nasa. gov/Library/Measuring-Vegetations.

68. Weiss, A. 2001. Topographic position and landforms analysis. In Poster presentation, ESRI user conference, San Diego, CA(Vol. 200).

69. Wilson, J.P. and J.C. Gallant. (Eds). 2000. Terrain analysis. Principles and applications. John Wiley \& Sons, New York.

70. www.usgs.com

71. Yilmaz, I. 2009. Landslide susceptibility mapping using frequency ratio, logistic regression, artificial neural networks and their comparison: a case study from Kat landslides (Tokat-Turkey).Computers \& Geosciences, 35(6): 1125-1138.

72. Zare, M., M.H. Jouri, D. Asharizadeh, T. Salarian and M. Fakhre Ghazi. 2016. An Evaluation of Landslide Hazard in Masooleh Watershed using Dempster- Shafer Theory and GIS, Journal of Watershed Management Research, 7(13): 209-217 (In Persian).

73. Zevenbergen, L.W. and C.R. Thorne. 1987. Quantitative analysis of land surface topography.Earth surface Processes and Landforms, 12(1): 47-56. 


\title{
Prioritization of Landslide Effective Factors using Logistic Regression (Case Study: A Part of KopeDagh-Hezar Masjed Zone)
}

\section{Shiva Abdi Bastami ${ }^{1}$, Hadi Memarian ${ }^{2}$, Seyed Mohammad Tajbakhsh ${ }^{2}$ and Mahmood Azami Rad $^{3}$}

1- M.Sc. Student, Department of Watershed Management Engineering, Faculty of Natural Resources, University of Birjand (Corresponding author: Bastamish22@gmail.com)

2- Assistant Professor, Department of Watershed Management Engineering, Faculty of Natural Resources, University of Birjand

3- Assistant Manager of Watershed Management, Natural Resources and Watershed Management Department of Khorasan Razavi Province

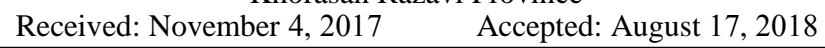

\begin{abstract}
Landslideeventin Iran, as a natural hazard, causes a lot of mortal and financial losses annually. Thus, the comprehensive researches are necessary to provide useful solution for preventing and reducing the damages caused by landslide events. Accordingly, the present study uses a logistic regression approach whit the aim of prioritizing the factors affecting the occurrence of landslides in a part of KopetDagh-HezarMasjed zone, in North-East of Iran. In this work, after applying the GIS functions on each initial information layer,the logistic regression analysis was performed to identify the effective factors, and then the frequency of landslides in each class was determined. Based on the results of Type II analysis, among all selected factors, soil factor with the lowest value of Pr>LR ratio (0.001) was identified as the most important driving force in landslide happening. After that, altitude, lithology, Topographic Position Index (TPI), Stream Power Index (SPI),Normalized Difference Vegetation Index (NDVI), roughness index and landuse, with the Pr>LR values of $0.002,0.003,0.004,0.032$, $0.037,0.100$, and 0.109 , had the most impact on the occurrence of landslides in the study area, respectively. Four initial factors at the $99 \%$ confidence level and other factors at $95 \%$ confidence level were effective in landslide occurrence in the study area.
\end{abstract}

Keywords: Geographic Information System (GIS), Landslide, Logistic Regression, Prioriz 